\title{
Kopulasätze als Zustandsbeschreibungen ${ }^{*}$
}

\author{
Johannes Dölling \\ Universität Leipzig \\ doelling@rz.uni-leipzig.de
}

\section{Einführung}

Auf die Frage, ob das Davidsonsche Herangehen auch auf Sätze mit der Kopula sein (resp. to be) ausgedehnt werden sollte und wenn ja, wie dies $\mathrm{zu}$ geschehen habe, gibt es unter den Vertretern der ereignisbasierten Semantik divergierende Antworten. Der Standpunkt, daß mit Kopulasätzen auf Instanzen einer eigenständigen ontologischen Kategorie, nämlich auf Zustände Bezug genommen wird, findet sich erstmalig in Parsons (1980). Ausführlicher wird für diese Position in Parsons (1990) und Parsons (1995) argumentiert, wobei allerdings im Falle von Kopulasätzen mit einem nominalen Prädikativ die klassische (und damit 'zustandsfreie') semantische Analyse als eine zu bevorzugende Option beibehalten wird. Dagegen müssen nach Bierwisch (1988) und Kamp \& Reyle (1993) beliebige der hier interessierenden Sätze so verstanden werden, daß man mit ihnen auf Zustände referiert. Eine derart einheitliche Behandlung von Kopulasätzen wiederum lehnen die Verfechter der Konzeption eines Stadien/Individuen-Konstrastes ausdrücklich ab. Gemäß Kratzer (1989) und Chierchia (1995) sind nur Sätze, in denen sogenannte Stadienausdrücke als Prädikative fungieren, als Beschreibungen von (temporären) Zuständen aufzufassen, während alle anderen Fälle im Sinne der klassischen Analyse als Zuweisungen einer (mehr oder weniger permanenten) Individueneigenschaft zu verstehen sind. In radikaler Abkehr von diesen Vorschlägen schließlich wird in Maienborn (1998) und Maienborn (1999) überhaupt bezweifelt, daß sich Kopulasätze auf Zustände (oder andere Situationen) beziehen können. Als deren Inhalt wird stattdessen im Kern eine Aussage über ein Zeitintervall angesehen, innerhalb dessen dem jeweiligen Individuum die durch das Prädikativ denotierte Eigenschaft zukommt.

Im vorliegenden Beitrag plädiere ich für ein Vorgehen, bei dem Kopulasätze generell als Beschreibungen von Situationen behandelt werden. Genauer nehme ich an, daß Sätze mit der Kopula sein semantische Repräsentationen haben, die über eine darin vorkommende existenzquantifizierte Variable auf eine noch näher zu spezifizierende Situation referieren. Drei grundlegende Klassen von Fällen werden unterschieden: Erstens kann es sich bei der fraglichen Situation um einen durch das Prädikativ charakterisierten Zustand handeln, in dem sich das mit dem Subjektausdruck erfaßte Objekt befindet. Zweitens kann die Situation ein mit dem Subjektausdruck erfaßter Zustand sein, der über das Prädikativ eine zusätzliche Charakterisierung erhält. Und drittens kann die Situation auch ein Ereignis (im weiteren Sinne) sein, das nun entsprechend mit dem betreffenden Subjektausdruck erfaßt und durch das Prädikativ näher charakterisiert wird. In meiner Darstellung werde ich mich auf die in der ersten Klasse zusammengefaßten Fälle des Zustandsbeschreibung konzentrieren. Dies nicht nur deshalb, weil offensichtlich die meisten Vorkommen von Kopulasätzen von dieser Art sind, sondern auch, weil mit ihnen - das zeigen die bisherigen Diskussionen - der größte Teil der Probleme verbunden ist. Was den Anteil der Kopula an der Bedeutung der Sätze betrifft, so gehe ich davon aus, daß die Einheit sein nichts zur semantischen Repräsentation des jeweiligen Prädi-

\footnotetext{
* Ich danke Manfred Bierwisch, Gerhard Jäger, Ewald Lang, Claudia Maienborn, Arnim von Stechow, Renate Steinitz und Ilse Zimmermann für kritische Hinweise, die sie mir anläßlich von Diskussionen über die in diesem Beitrag vorgetragenen Ideen gaben. Die Arbeit entstand im Rahmen des von der DFG geförderten Projekts "Schnittstellen der Semantik: Prädikativkonstruktionen" am ZAS Berlin.
} 
Auf dem Hintergrund dieser Kriterien gibt es Indizien dafür, daß beliebige Kopula-PrädikativKonstruktionen wie glücklich sein, ein Künstler sein oder auf der Straße sein gemeinsam mit Verben wie lieben, ähneln oder besitzen zur Klasse der stativen Verb(alphras)en gehören. Bei der gegebenen Verbklassifizierung handelt es sich allerdings um eine Unterscheidung auf rein sprachlicher Ebene. Nichts ist damit darüber gesagt, ob die auf diese Weise herausgehobenen Ausdrücke auch passende Korrelate in der Welt besitzen, d.h. ob sie von Entitäten prädiziert werden, die man als eigenständige ontologische Sorte unter der Bezeichnung Zustand zusammenfassen kann. Hierfür müssen zusätzliche Daten herangezogen werden.

Welche Evidenzen lassen sich also für den Zustandsbezug von Kopulasätzen finden? Welche sprachlich begründete Rechtfertigung gibt es damit für die Annahme von Zuständen in der Ontologie der Commonsense-Welt? Zur Beantwortung dieser Fragen bietet es sich an, auf Kriterien zurückzugreifen, die in der Ereignissemantik als Argumente einer Reifizierung von Ereignissen und Prozessen benutzt werden.

\section{(A) Anwendung von Modifikatoren:}

Einwandfreie Beispiele für Sätze, in denen die Kopula-Prädikativ-Konstruktion durch ein adverbiales Adjunkt modifiziert und dabei unmittelbar ein zugrundeliegender Zustand spezifiziert wird, sind nicht einfach anzugeben. ${ }^{4}$ Wie vor allem in Maienborn (1999) überzeugend gezeigt wird, darf zunächst einmal das Vorkommen von Lokaladverbialen nicht als Indiz dafür angesehen werden, daß der jeweilige Kopulasatz auf einen Zustand referiert. ${ }^{5}$ Adverbiale wie auf der Straße und im Kino in (1a) bzw. in (1b) sind in keinem Fall an die jeweiligen VPn adjunktiert; sie fungieren deshalb auch nicht als zustandsbezogene, sondern als sogenannte rahmensetzende Modifikatoren.

(1) (a) Hans war auf der Straße betrunken.

(b) Maria war im Kino ohnmächtig.

Dagegen handelt es sich bei den Temporaladverbialen einen Tag lang und seit zehn Minuten in (2a) bzw. (2b) eindeutig um Adjunkte, die die vorkommenden Kopula-PrädikativKonstruktionen modifizieren.

(2) (a) Hans war einen Tag lang betrunken.

(b) Maria war seit zehn Minuten ohnmächtig.

Es ist keineswegs so, daß man solche Verbindungen für sich genommen schon als schlagendes Argument für den Zustandsbezug von Kopulasätzen akzeptieren muß, da ja die betreffenden temporalen Adverbiale im gegebenen Zusammenhang einfach als Prädikate von Zeitintervallen angesehen werden könnten. ${ }^{6}$ Die Sätze bezögen sich dann auf entsprechend näher spezifizierte Zeiten, zu denen die mit den Subjekten denotierten Objekte die jeweiligen Eigenschaften haben. Im Kontext der in ihrer Gesamtheit zu berücksichtigenden sprachlichen Daten erscheint mir diese Option jedoch als wenig überzeugend. Was mit temporalen Modifikatoren wie den angegebenen ausgedrückt werden soll, ist stattdessen eine zeitliche Charakterisierung

\footnotetext{
4 Zur herausragenden Rolle, die adverbielle Modifikationen für die Begründung eines ereignisbasierten Herangehens spielen, siehe Eckardt (1998a).

s Einige Autoren (siehe z. B. Kratzer 1989 und Chierchia 1995) betrachten gerade lokale - neben temporalen Modifikatoren als einen Beleg für den Situations-(resp. Zustands-)bezug von Kopulasätzen.

6 Diese Haltung wird angesichts der mit (1a) und (1b) exemplifizierten Unzulässigkeit lokaler Modifikation in Maienborn (1998) und Maienborn (1999) unter der m.E. unbegründeten Voraussetzung eingenommen, daß die räumliche Bestimmung ein notwendiges Kennzeichen von Zuständen ist. Zur Möglichkeit, Ereignisse (im weiteren Sinne) als Zeitintervalle zu rekonstruieren, siehe auch Eckardt (1998b).
} 
jener Zustände, in denen die betreffenden Objekte sind. Demnach drückt (2a) aus, daß Hans sich im Zustand des Betrunkenseins befand und dieser Zustand einen Tag lang dauerte, und (2b) referiert auf einen Zustand, bei dem es sich um ein - von einem Bezugspunkt aus gesehen - seit zehn Minuten andauerndes Ohnmächtigsein von Anna handelte.

Auch die Sätze in (3) könnten so verstanden werden, daß mit ihnen das erneute Vorhandensein eines Zeitintervalls konstatiert wird, innerhalb dessen Anna glücklich oder Ehefrau oder in der Stadt war - dies als Umschreibung der sog. restitutiven Interpretation von wieder.

Anna war wieder glücklich / Ehefrau / in der Stadt.

Doch ebenso wie bei den noch zu betrachtenden Fällen der Modifikation denke ich, daß eine solche Sichtweise zu kurz greift. Nicht zuletzt mit Blick auf die restitutive Verwendung von wieder in nicht-stativen Sätzen halte ich jene Interpretation für intuitiv angemessener, wonach hier ausgedrückt wird, daß sich Anna zum wiederholten Male in Zuständen der beschriebenen Art befand. In (3) tritt damit das Adverb wieder nicht als ein Prädikat von Zeitintervallen, sondern von Zuständen auf. Analoges gilt für das Vorkommen von Frequenzadverbialen in Kopulasätzen wie in (4).

(4) Peter war dreimal krank / der Sieger / an der Spitze.

Durch Modifikation der jeweiligen Kopula-Prädikativ-Konstruktion mit dem Adverb dreimal wird die Anzahl der betreffenden Zustände, nicht jedoch - oder aber höchstens mittelbar - die Anzahl der entsprechenden Zeitintervalle spezifiziert.

Während komitative und instrumentale mit/ohne-PPn als Adjunkte in Sätzen mit Adjektivoder DP-Prädikativen ausgeschlossen zu sein scheinen, ist ihre Adjunktion an die jeweilige VP dann völlig akteptabel, wenn wie in (5a) und (5b) eine lokale PP als Prädikativ fungiert.

(5) (a) Sebastian war ohne / mit Maria im Zimmer.

(b) Hans war ohne / mit dem Auto in der Stadt.

Obwohl die Adverbiale in beiden Fällen Prädikate von Zuständen sind, gibt es dennoch einen wichtigen Unterschied zwischen ihnen. In (5a) wird wieder unmittelbar ein Zustand, genauer: Sebastians Zustand des Im-Zimmer-seins, mit Hilfe der Modifikatoren ohne/mit Maria charakterisiert. ${ }^{7}$ Dagegen wird in (5b) anders als bei den bisherigen Beispielen durch die Adverbiale ohne/mit dem Auto nicht unmittelbar der beschriebene Zustand, sondern jenes Ereignis näher spezifiziert, dessen Resultat er ist. Auch die in (6a) - (6c) verwendeten modalen Adverbien schnell, plötzlich und absichtlich sind offensichtlich Modifikatoren, die eine derart vermittelte Evidenz für die Zustandsreferenz der betreffenden Sätze liefern.

(6) (a) Fabio war schnell reich / im Theater / ein berühmter Künstler.

(b) Claudia war plötzlich wach / am Fenster / eine Millionärin.

(c) Peter war absichtlich betrunken / in der Kneipe / der Verlierer.

Während in (6a) ausgedrückt wird, daß die betreffenden Zustände das Ergebnis von jeweils schnellen Veränderungen waren, werden die in $(6 \mathrm{~b})$ erwähnten Zustände dadurch indirekt charakterisiert, daß ihr Eintreten als plötzlich spezifiziert wird. Mit (6c) schließlich wird zu erkennen gegeben, daß die Peter eigenen Zustände aus Ereignissen resultierten, die von ihm

\footnotetext{
Es sei betont, daß eine genauere Analyse von Sätzen wie (5a) sicher komplexere Zusammenhänge zu berücksichtigen hat, als dies im gegebenen Rahmen geschehen kann.
} 
mit Absicht realisiert worden waren. Um wiederum andere Fälle des Zustandsbezugs von Modifikatoren handelt es sich bei den in (7a) und (7b) vorkommenden modalen PPn mit/ohne Begeisterung bzw. Leidenschaft.

(7) (a) Renate war mit / ohne Begeisterung Lehrerin.

(b) Hans war mit / ohne Leidenschaft Angler.

Hier erfahren die Zustände des Lehrerinseins von Renate und des Anglerseins von Hans dadurch eine Charakterisierung, daß die Einstellungen dieser Personen zu den mit den Zuständen jeweils verbundenen Tätigkeiten spezifiziert werden.

(B) Anaphorische Beziehungen:

Ebenso wie beim vorangehend betrachteten Datenbereich kostet es auch hier einige Mühe, eindeutige Hinweise auf einen Zustandsbezug von Kopulasätzen $\mathrm{zu}$ finden. Offensichtlich kann an einen Satz wie (8a) jeder der in (8b) - (8d) aufgeführten Sätze angeschlossen werden.

(8) (a) Anna war verheiratet.

(b) Das war eine ereignisreiche Zeit.

(c) Das war ein nur kurzzeitiger Zustand.

(d) Das ist ein bekannter Sachverhalt.

Damit scheint das anaphorische Pronomen das jeweils einen anderen - in (8a) implizit gegebenen - Antezedenten zu haben. Im Falle von (9a) - (9c) referiert die Anapher in (9b) ganz sicher nicht auf den Sachverhalt, auf den sich Satz (9a) bezieht.

(9) (a) Renate war krank.

(b) Das dauerte drei Wochen.

(c) *Dieser Sachverhalt / dieser Zustand / diese Zeit dauerte drei Wochen.

Dies geht explizit aus (9c) hervor und stimmt mit der gängigen Auffassung überein, wonach Sachverhalte als abstrakte Gegenstände keiner zeitlichen Bestimmung unterliegen. Wie (9c) außerdem zeigt, ist aber sowohl eine Bezugnahme auf den jeweiligen Zustand als auch auf das zugeordnete Zeitintervall zugelassen. Anders verhält es sich hingegen im folgenden Fall:

(10)(a) Claudia war glücklich.

(b) Das wurde von allen bemerkt.

(c) Dieser Sachverhalt / dieser Zustand / *diese Zeit wurde von allen bemerkt.

Weil im gegebenen Zusammenhang zwar von Sachverhalten oder von Zuständen, aber nicht oder zumindest nicht im wörtlichen Sinne - von Zeitintervallen gesagt werden kann, daß sie bemerkt wurden, ist lediglich eine Referenz auf die durch (10a) erfaßten Instanzen der beiden anderen Arten möglich. Unter Berücksichtigung dieser Überlegungen läßt sich dann ein Beispielpaar wie (11a)/(11b) als ein Beleg für den Zustandsbezug von Kopulasätzen und gegen Versuche einer Reduktion von Zuständen auf Zeitintervalle oder Sachverhalte anführen.

(11)(a) Paul war betrunken.

(b) Das / dieser Zustand dauerte zwei Tage und wurde von allen bemerkt.

Nur auf Zustände ist offensichtlich ein Prädikat anwendbar, das aus der Koordination von VPn wie dauerte zwei Tage und wurde von allen bemerkt entsteht. 
(C) Wahrnehmungssätze:

Wie aus (12a) - (12c) hervorgeht, können Kopula-Prädikativ-Konstruktionen nicht in Infinitivkomplementen von Wahrnehmungsverben auftreten.

(12)(a) *Maria sah Hans nackt / wütend / betrunken / krank / müde sein.

(b) *Fabio sah Peter auf der Straße/ mit Mantel / in Not sein.

(c) *Sebastian sah Anna eine Patientin / Ehefrau / die Siegerin sein.

Damit ist die für die ereignisbasierte Analyse wichtige Erkenntnis, wonach Wahrnehmungssätze dieser Form ausdrücken, daß vom Subjekt eine durch das Infinitivkomplement bezeichnete Situation wahrgenommen wird, für die hier verfolgten Zwecke wenig hilfreich. Vielmehr scheint die Unzulässigkeit von Sätzen wie in (12a) - (12c) gerade gegen die Annahme zu sprechen, daß Kopulasätze auf Zustände referieren.

Es gibt aber auch Wahrnehmungssätze, die wenigstens eine indirekte Evidenz für den Zustandsbezug von (bestimmten) Kopulasätzen liefern. ${ }^{8}$ Diese unterscheiden sich von Sätzen der oben angegebenen Art lediglich dadurch, daß die Kopula, die ja in ihrer infiniten Form ohnehin keine Funktion als Tempusträger zu erfüllen hat, im Komplement der Wahrnehmungsverben nicht enthalten ist. Betrachten wir dazu die in (13a) - (13c) angeführten Sätze.

(13)(a) Maria sah Hans nackt / wütend / betrunken / *krank / *müde.

(b) Fabio sah Peter auf der Straße / mit Mantel / in Not.

(c) *Sebastian sah Anna eine Patientin / Ehefrau / die Siegerin.

Bezogen auf (13a) und (13b) zeigt sich, daß nunmehr die meisten der als Beispiel benutzten Sätze akzeptabel sind. Bedeutsam ist dabei, daß die betreffenden Sätze - bis auf den ersten Satz von (13b) sogar in präferierter Lesart ${ }^{9}$ - als Beschreibungen von Situationen verstanden werden können, in denen das Subjekt jene Zustände sah, auf die mit den jeweiligen Komplementen des Verbs Bezug genommen wird. Danach besagt beispielsweise Maria sah Peter nackt nicht etwa, daß von Maria der nackte Peter gesehen wurde. Sicher soll auch nicht ausgedrückt werden, daß sie den Sachverhalt sah, daß Peter nackt war. Schon gar nicht kann Inhalt des Satzes sein, daß Maria eine Zeit des Nacktseins von Peter sah. Letzteres ist ja schon wegen der Nichtwahrnehmbarkeit von Zeitintervallen ausgeschlossen. Was mit dem Satz ausgedrückt wird, ist vielmehr, daß Gegenstand des Sehens von Maria ein Zustand der Nacktheit von Peter war.

Aus der Beobachtung, daß sich die in Wahrnehmungssätzen als Verbkomplement auftretenden kopulafreien Konstruktionen auf Zustände beziehen, lassen sich zwei wichtige Folgerungen für die weitere Analyse gewinnen: Erstens ist damit belegt, daß auch entsprechende Kopulasätze mit den Prädikativen nackt, wütend, betrunken, auf der Straße usw. auf Zustände referieren. Zweitens wird indiziert, daß die Kopula selbst gar keinen Beitrag zum Zustandsbezug von Kopulasätzen leistet. Was dann noch offen bleibt, ist zu erklären, warum nicht belie-

\footnotetext{
8 Vgl. Parsons $(1990,193)$ und Chierchia $(1995,178)$.

Die näherliegende Lesart von Fabio sah Peter auf der Straße ist sicher die, bei der das Sehereignis auf der Straße lokalisiert wird. Auch für den Satz Fabio sah Peter mit Mantel könnte man sich eine Interpretation vorstellen, wo mit Mantel als ein Modifikator der VP fungiert. Für alle Sätze ist schließlich die m.E. wenig wahrscheinliche Lesart möglich, in der durch nackt, wütend usw. das Subjekt näher spezifiziert wird.
} 
bige Adjektive und warum überhaupt keine DPn in den fraglichen Komplementen von Wahrnehmungsverben vorkommen dürfen. ${ }^{10}$

(D) Nominalisierungen:

Aus Adjektiven und PPn lassen sich mit Hilfe der Kopula Konstruktionen wie Nacktsein, Betrunkensein, In-der-Stadt-sein oder In-Not-sein gewinnen. Soweit man Ausdrücke dieses Typs als abgeleitete Nomina betrachten will, hat man es dabei mit Bezeichnungen für Zustände und nicht etwa für Zeitintervalle zu tun. Allerdings erscheinen derartige Konstruktionen als recht unnatürlich und werden vorrangig als eher technische Mittel des Zustandsbezugs benutzt. Die allgemein gebräuchliche Form der Nominalisierung von Adjektiven ist jene unter Verwendung von Suffixen, zu denen vor allem -heit und -keit gehören. Im Falle von abgeleiteten Nomina wie Nacktheit, Schönheit, Ähnlichkeit, Abhängigkeit, Stille, Kälte, Eleganz oder Ignoranz handelt es sich offensichtlich ebenfalls um Prädikate von Zuständen (mit gegebenenfalls unterschiedlicher prototypischer Dauer). Während der Inhalt von DPn wie Marias Schönheit, Fabians Ähnlichkeit mit Hans oder Claudias Eleganz in bestimmten Kontexten vielleicht auch mit Sachverhaltsbestimmungen wie daß Maria schön ist etc. wiedergegeben werden kann, scheint es dagegen unmöglich zu sein, daß man sich mit ihnen auf Zeitintervalle bezieht. Worauf die gegebenen Ausdrücke normalerweise referieren, sind die entsprechenden Zustände von Maria, Fabian und Claudia. Beispiele, bei denen auf Grund der Kombination der Prädikate ein Bezug auf einen Sachverhalt oder ein Zeitintervall eindeutig ausgeschlossen und deshalb die Zustandsreferenz unabdingbar ist, liefern (14a) - (14b).

(14) (a) Die wochenlange Krankheit von Anna beunruhigte alle.

(b) Sebastians Abhängigkeit war dauerhaft und folgenreich.

(c) Die bedrückende Stille währte einige Minuten.

Unter der Voraussetzung, daß die Quelle der konstatierten Eigenschaft nicht erst in der Nominalisierung ${ }^{11}$, sondern - wie schon im Zusammenhang mit Wahrnehmungssätzen nahegelegt in den zugrundeliegenden Adjektiven zu sehen ist, besitzen wir damit ein zusätzliches Indiz für die Richtigkeit der hier verfochtenen Annahme.

Insgesamt erfährt so die Behauptung, daß Kopulasätze auf Zustände referieren, durch unterschiedliche Daten eine direkte oder zumindest indirekte Stützung. Weitere Argumente, mit denen sich diese Feststellung theoretisch untermauern läßt, werde ich im folgenden liefern.

\section{Zustandsprädikate und die Kopula}

Wie nun sind Kopulasätze genauer zu analysieren? Ist bei allen Kopulasätzen vom Bezug auf einen Zustand und in diesem Sinne vom Vorhandensein einer existentiell quantifizierten Zustandsvariablen in deren Bedeutungsstruktur auszugehen? Wird die betreffende Zustandsvariable durch die Kopula in die semantische Repräsentation eingebracht oder resultiert sie vielmehr aus Bedingungen, die mit der Bedeutung der jeweiligen Prädikative zusammenhängen? Anhand einer kritischen Beleuchtung der mit verschiedenen Konzeptionen formulierten Antworten sowie den sich daraus ergebenden Konsequenzen sollen in diesem Abschnitt die

\footnotetext{
10 Im Falle von Adjektiven nehme ich eine konzeptuelle Bedingung derart an, daß die jeweiligen Zustände ein bestimmtes $\mathrm{Maß}$ an Sichtbarkeit haben müssen, um zum Gegenstand des Sehens werden zu können. Dagegen ist es möglich, daß sich die generelle Unzulässigkeit von DPn in solchen Konstruktionen aus syntaktischen Beschränkungen ergibt.

"Beispielsweise nimmt Zimmermann (1996) an, daß das Zustandsargument über den Bedeutungsbeitrag des jeweiligen Suffixes in die semantische Struktur des Nomens eingeführt wird.
} 
Grundzüge meines Verständnisses von Kopulasätzen als Zustandsbeschreibungen entwickelt werden.

Wahrscheinlich nicht zuletzt mit Blick auf die ihnen zugewiesene Rolle als Tempusträger wird in Bierwisch (1988) und in Kamp \& Reyle (1993) für alle Verben und damit auch für die Kopula davon ausgegangen, daß sie in ihrer lexikalisch-semantischen Repräsentation über ein Situationsargument verfügen. Genauer wird von diesen Autoren vorausgesetzt, daß die semantische Funktion der Kopula gerade darin besteht, Prädikativen wie kalt, ein Lehrer oder ohne Hoffnung ein Zustandsargument zuzuordnen. Nur in Gestalt dieser Zustandsvariablen weisen Kopula-Prädikativ-Konstruktionen überhaupt eine Argumentstelle auf, an die bei adverbiellen Modifikationen und bei der Tempusspezifizierung des jeweiligen Satzes angeknüpft werden kann. So nimmt Bierwisch (1988) an, daß das Lexem sein die in (15) aufgeführte semantische Struktur hat.

$$
\lambda \mathrm{P} \lambda \mathrm{u} \lambda \mathrm{s} . \mathrm{s} \operatorname{INST}[\mathrm{P}(\mathrm{u})]
$$

(mit u und s als Variablen für Objekte bzw. für Situationen oder Zustände)

Die Bedeutung eines Satzes wie (16) kann dann - bei Ausblendung von Tempus und anderer hier irrelevanter Faktoren - entsprechend durch (16') repräsentiert werden.

Die Wohnung war zwei Tage lang kalt.

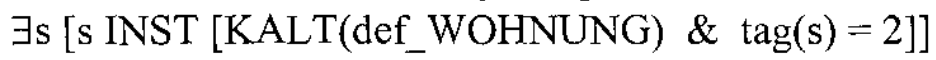

Mit dieser wie auch entsprechend mit der in Kamp \& Reyle (1993) vorgeschlagenen Herangehensweise sind aber mindestens zwei Schwierigkeiten verbunden. Erstens haben wir nun zwar eine Erklärung dafür, wie ein temporaler Modifikator auf eine Kopula-PrädikativKonstruktion angewandt werden kann, unklar bleibt dabei aber dessen Anwendung im Rahmen von Adjektivphrasen wie in (17).

$$
\text { Maria war in einer zwei Tage lang kalten Wohnung. }
$$

Da das Adjektiv kalt hier ein Prädikat von Objekten ist und deshalb über kein Argument verfügt, das durch zwei Tage lang näher spezifiziert werden kann, scheint es unter den gegebenen Voraussetzungen nur zwei Wege zu geben, um die Modifikation zu realisieren. Zum einen könnte angenommen werden, daß beim Adjektiv durch eine zusätzliche, an der Oberfläche nicht sichtbare Operation das erforderliche Zustandsargument eingeführt wird. Zum anderen wäre es möglich, für solche Fälle einfach ein anderes Verständnis von Modifikation zugrunde $\mathrm{zu}$ legen. Es ist offensichtlich, daß beide Lösungsvorschläge wegen ihres ad-hoc-Charakters ausscheiden. ${ }^{12}$ Ein zweites Problem, mit dem wir konfrontiert werden, ergibt sich aus Beispielen wie (18).

$$
\text { Es war zwei Tage lang kalt. }
$$

Nimmt man für die Kopula eine Bestimmung wie die in (15) an, so bleibt bei Sätzen, in denen Kopula-Prädikativ-Konstruktionen ohne Subjekt verwendet werden, die für das Subjekt vorgesehene Argumentstelle ungesättigt. Ein überzeugender Vorschlag, der auch die Parallelität

12 Eine analoge Schwierigkeit ergibt sich selbstverständlich auch bei dem in Maienborn (1999) gemachten Vorschlag, die Repräsentation in (15) durch die folgende zu ersetzen:

(i) $\lambda \mathrm{P} \lambda \mathrm{u} \lambda \mathrm{t}$. $[\mathrm{P}(\mathrm{u})] \mathrm{AT} \mathrm{t}$

Hier muß dann beispielsweise auf irgendeine Weise ein Zeitargument in die semantische Struktur eingebracht werden. 
eines solchen Satzes zu Beispielen wie Es regnete berücksichtigt, existiert bisher ebensowenig wie im Falle der ersten Schwierigkeit.

Ein erstes Fazit unserer Betrachtungen ist, daß die Idee, die Kopula zum Lieferanten der Zustandsvariablen zu machen, als empirisch wenig begründet angesehen werden muß. Wie sich anhand des mit (17) exemplifizierten Problems gezeigt hat, ist zumindest bei Adjektiven wie kalt, krank, glücklich oder müde vom Vorhandensein eines Zustandsarguments auszugehen. Die einzig mögliche Alternative scheinen deshalb Ansätze zu sein, bei denen die Quelle des Zustandsarguments nicht in der Kopula, sondern im Prädikativ angesiedelt wird.

Betrachten wir zunächst Vorschläge, die unter der Voraussetzung eines Kontrastes zwischen Individuen- und Stadienprädikaten gemacht worden sind. Nach Kratzer (1989) unterscheiden sich Stadienprädikaten von Individuenprädikaten dadurch, daß sie zusätzlich zu der für Objekte reservierten Argumentposition mit einem sogenannten Davidsonschen Argument ausgestattet sind und damit eine raum-zeitliche Relativierung der jeweiligen Eigenschaft kenntlich machen sollen. In Abweichung von der Standardauffassung kann man demnach beispielsweise für müde die lexikalisch-semantische Repräsentation (19) annehmen, wodurch als Denotat des Adjektivs eine Relation zwischen Situationen s und Objekten u ausgewiesen wird.

$$
\lambda u \lambda s . M U ̈ D E(u)(s)
$$

Während Sätze, deren Prädikative von Individuenprädikaten wie blond oder Lehrer gebildet werden, weiterhin Repräsentationen im traditionellen Format haben, erhält dann ein Satz wie (20) eine Repräsentation, aus der dessen Situationsreferenz hervorgeht.

Anna war müde.

(20') $\exists$ s [MÜDE(anna)(s)]

Im Rahmen eines solchen Verständnisses kann man unterschiedliche Vorstellungen vom Bedeutungsgehalt der Kopula entwickeln. So könnte angenommen werden, daß die Kopula einfach die semantische Repräsentation (21) aus dem Lexikon mitbringt und damit deren Beitrag zur Bedeutung von entsprechenden Sätzen gleich Null wäre.

$$
\lambda \text { P. P }
$$

(mit P als Variable für beliebige Prädikate der 1. Stufe)

Ein Mangel des Herangehens ist, daß nun zwar bei Sätzen mit Stadienprädikaten, nicht aber bei solchen mit Individuenprädikaten ein Ansatzpunkt für die Tempusbestimmung des jeweiligen Satzes vorhanden ist. ${ }^{{ }_{3}}$ Denkbar wäre es, diese Schwierigkeit dadurch zu umgehen, daß die Kopula als polysem behandelt wird und dementsprechend für sie zwei Repräsentationen eine zur Anwendung auf Stadienprädikate und eine zur Anwendung auf Individuenprädikate vorgesehen werden. Anders als bei der Verbindung mit einem Stadienprädikat könnte dann die Kopula (etwa unter Voraussetzung einer Repräsentation wie der in Anmerkung 12 angegebenen) bei Individuenprädikaten eine entsprechende Anreicherung der Argumentstruktur herbeiführen. Problematisch an einem solchen Vorschlag ist aber nicht nur die m.E. unbegründete Annahme einer Mehrdeutigkeit der Kopula. Verschärft wird durch ihn vielmehr auch Kratzers Dilemma, daß sie nämlich einerseits zwar die Möglichkeit einer Transformation von

\footnotetext{
13 Kratzers eigene Idee, wonach das Tempus eines Satzes mit einem Individuenprädikat als Angabe der Existenzzeit des jeweiligen Objektes verstanden werden kann, hält einer kritischen Betrachtung nicht stand. Wie auch in Musan (1997) gezeigt wird, kann natürlich aus einem Satz wie Anna war blond keineswegs logisch gefolgert werden, daß Anna inzwischen gestorben ist.
} 
Individuenprädikaten in Stadienprädikate und umgekehrt einräumt, andererseits aber nicht genauer angibt, wie und unter welchen Bedingungen eine solche Transformation erfolgt. ${ }^{14}$

Eine partielle Lösung des vorangehend erwähnten Problems bietet Chierchia (1995) an. Anders als in Kratzer (1989) wird zunächst einmal angenommen, daß beliebige Adjektive und Nomina über ein Situationsargument verfügen. ${ }^{15}$ So gesehen haben jetzt nicht nur krank oder glücklich, sondern auch blond, intelligent, Lehrerin oder Franzose semantische Repräsentationen analog zu der für müde in (19) und sind damit Stadienprädikate. Daneben können nach Chierchia aber beliebige Adjektive und Nomina auch als Individuenprädikate verwendet werden. Ihre interne semantische Struktur ist dann dadurch bestimmt, daß das in den jeweiligen Stadienprädikaten vorkommende Situationsargument auf passende Weise durch einen generischen Operator abgebunden ist. So hat beispielsweise das Nomen Koch die beiden in (22a) und (22b) angegbenen semantischen Repräsentationen.

\section{(22)(a) $\lambda u \lambda s . \operatorname{KOCH}(u)(s)$ \\ (b) $\lambda \mathrm{u} . \mathrm{KOCH}^{*}(\mathrm{u})$ $=\lambda \mathrm{u} . \mathrm{GENs}[\mathrm{IN}(\mathrm{s})(\mathrm{u})][\mathrm{KOCH}(\mathrm{u})(\mathrm{s})]$}

Als Stadienprädikat denotiert Koch demnach eine Relation zwischen Situationen s und Objekten $\mathrm{u}$; als Individuenprädikat steht es dagegen für eine Eigenschaft von Objekten $u$ derart, daß $u$ typischerweise in Situationen, in denen es lokalisiert ist, ein Koch ist. Ein Nomen ist dabei genau dann ein bereits im Lexikon abgeleitetes Individuenprädikat, wenn es nicht-prädikativ gebraucht wird. Bei prädikativer Verwendung werden Nomina dagegen erst auf der syntaktischen Ebene in Individuenprädikate überführt, um temporale und adverbielle Bestimmungen zu ermöglichen. Analoge Annahme werden für Adjektive gemacht.

Eine abschließende Bewertung der in Chierchia (1995) formulierten Ideen kann im gegebenen Rahmen nicht vorgenommen werden. Dies ist unter anderem auch deshalb unmöglich, weil die dort - häufig als gegeneinander austauschbar - verwendeten Ausdrücke Situation und $\mathrm{Zu}$ stand hinsichtlich ihrer Bedeutung $\mathrm{zu}$ unbestimmt sind. ${ }^{16}$ Wenn von der Lokalisierung von Objekten in Situationen gesprochen wird, muß man davon ausgehen, daß damit wahrscheinlich nicht das Befinden der Objekte in Zuständen derart gemeint ist, daß die ersteren Inhaber der letzteren sind. Intuitiv unangemessen ist $\mathrm{m}$.E. an den Vorschlägen, daß Nomina erst in einem abgeleiteten Sinne als Prädikate von Objekten auftreten sollen. Ganz offensichtlich spielen Objekte in unserer Konzeptualisierung der Commonsense-Welt insofern eine besondere Rolle, als sich mit ihnen gewöhnlich eine gewisses Maß an 'Kontinuität' verbindet. ${ }^{17}$ Der bevorzugte Sinn von Nomina sollte deshalb darin bestehen, solche Objekte als Instanzen von entsprechenden Konzepten auszuweisen. Umgekehrt ist zu überdenken, ob nicht auch Kopulasätze, in denen so genannte Individuenprädikate in prädikativer Position verwendet werden, als Beschreibungen von mehr oder weniger stabilen Zuständen der fraglichen Objekte zu verstehen sind. Welcher Unterschied wenn nicht der, daß sich Hans, Peter und Paul verschieden lang im Zustand des Kochseins befanden, sollte etwa mit (23a) - (23c) ausgedrückt werden?

\footnotetext{
14 Siehe hierzu u.a. die kritischen Bemerkungen in Maienborn (1996).

15 Dieselbe Voraussetzung findet sich beispielsweie auch bei Jacobs (1995) und bei Higginbotham \& Ramchand (1996), wo allerdings eine andere Position zur Stadien/Individuen-Distinktion eingenommen wird.

${ }_{16}$ Chierchia tut so leider nur wenig gegen die seit Kratzer (1989) bestehende Unsicherheit, wie denn genauer der von Kratzer gebrauchte Ausdruck Davidsonsches Argument verstanden werden soll. In Kratzer (1994) wird dagegen - offensichtlich unter dem Einfluß von Parsons (1990) - kenntlich gemacht, daß man es bei Adjektiven wie müde oder krank mit Zustandsargumenten $\mathrm{zu}$ tun hat.

i7 Siehe Simons (1987).
} 
(23)(a) Hans war sein Leben lang ein Koch

(b) Peter war eine längere Zeit ein Koch.

(c) Paul war nur fünf Minuten lang ein Koch.

Da ohnehin der angenommene Stadien/Individuen-Kontrast nicht nur erklärtermaßen kontextabhängig ist, sondern sich zunehmend auch das Fehlen einer grammatischen Basis für ihn zeigt, liegt es nahe, die damit angepeilten Unterscheidungen in den Bereich der konzeptuellen Interpretation einzuordnen. ${ }^{18}$

Nach dem bisher Gesagten kann man folgende Hypothesen formulieren: ${ }^{19}$

$$
\text { Beliebige Prädikative - seien sie nun nominaler, adjektivischer oder präpositionaler }
$$
Art - sind Prädikate von Zuständen.

Die Kopula ist für eine Anwendung auf Zustandsprädikate definiert und fügt nichts zu deren Bedeutung hinzu.

Die Hypothesen sollen zunächst mit Blick auf adjektivische Prädikative getestet werden, weil diese im Vergleich zu den übrigen Fällen relativ unproblematisch sind. Im Anschluß an Parsons (1990; 1995) will ich annehmen, daß Adjektive wie müde, krank, glücklich, blond oder intelligent lexikalisch-semantische Repräsentationen haben, die sie als Zustandsprädikate ausweisen. Beispielsweise ist mit krank die in (24) angegebenen Struktur verbunden.

$$
\lambda \text { s. KRANK(s) }
$$

Anders als in der Standardsemantik vorausgesetzt denotiert also krank keine Eigenschaft von Objekten, sondern von Zuständen, in denen sich Objekte befinden können. Für einen Kopulasatz wie (25) erhält man unter diesen Bedingungen - wiederum mit einigen Vereinfachungen - die semantische Repräsentation (25'), wobei HOLDER als Prädikat für die thematische Relation des Zustandsinhabers verwendet wird.

$$
\text { Renate war einen Monat lang krank. }
$$

$$
\exists \mathrm{s}[\operatorname{HOLDER}(\text { renate)(s) \& } \operatorname{KRANK}(\mathrm{s}) \& \operatorname{monat}(\mathrm{s})=1]
$$

Demnach referiert (25) auf einen (vergangenen) Zustand s derart, daß die Inhaberin von s Renate und s ein Zustand des Krankseins ist, der einen Monat lang dauert. Wie (26') zeigt, läßt sich nun eine NP, in der ein attributiv verwendetes Adjektiv mit Modfikator vorkommt, ganz analog behandeln.

zwei Jahre lang glückliche Frau $\lambda$ u. $\exists$ s $[\operatorname{HOLDER}(\mathrm{u})(\mathrm{s}) \&$ GLÜCKLICH(s) \& jahr(s) $=2] \&$ FRAU(u)

Und auch die semantische Repräsentation eines subjektlosen Satzes wie (27) stellt insofern kein Problem mehr dar, als jetzt die mit (27') gegebene Struktur angenommen werden kann. ${ }^{20}$

\footnotetext{
18 Siehe die detaillierten Erörterungen dazu in Jäger (1999) und Maienborn (1999).

19 Beide Hypothesen werden später unter anderem dahingehend zu präzisieren sein, daß auch Prädikate von Ereignissen und Prozessen als Prädikative auftreten können.

${ }^{20}$ Kopulasätze wie (27) scheinen damit einen Hinweis darauf zu liefern, daß es auch Zustände ohne Objekte als Inhaber gibt. Ich vermute allerdings, daß mit Blick auf Sätze wie In Berlin war es eine Woche lang warm eine Analyse erforderlich ist, bei der ein nicht näher spezifiziertes Objekt $u$ als Zustandsträger angenommen wird.
} 
Was aber noch nicht erklärt ist - und was Parsons schuldig bleibt -, ist das kompositionelle Zustandekommen der betreffenden Repräsentationen. Wie vor allem gelangt die HOLDERKomponente in die Bedeutungsstruktur von Konstruktionen wie (25) und (26)? Auf welcher Grundlage erfolgt jeweils die existentielle Abbindung der Zustandsvariablen in (25') - (27')? Auf diese und weitere Fragen werde ich im nächsten Abschnitt eine Antwort geben. An dieser Stelle sei lediglich die Struktur angegeben, die von mir der Kopula als (provisorische) lexikalisch-semantische Repräsentation zugewiesen wird.

$$
\lambda \mathrm{P} \lambda \text { s. } \mathrm{P}(\mathrm{s})
$$

Von der klassischen Version ${ }^{21}$ des lexikalischen Bedeutungseintrages für die Kopula unterscheidet sich (28) dabei lediglich dadurch, daß in Gestalt der s-Variablen die mit (II) formulierte semantische Anwendungseinschränkung auf Zustandsprädikate realisiert ist.

Betrachten wir nun Kopulasätze, in denen eine DP als Prädikativ fungiert. In Parsons (1990; 1995 ) wird es für möglich gehalten, Nomina wie Französin, Lehrer oder Baum in ihrer primären Bedeutung als Prädikate von Zuständen aufzufassen. Demnach hat etwa Französin die lexikalisch-semantische Repräsentation (29a). Zu Prädikaten von Objekten werden die betreffenden Nomina erst, wenn sie als Köpfe von nicht-prädikativ gebrauchten DPn auftreten. Thre Repräsentationen sind dann Abkürzungen nach dem in (29b) für Französin benutzten Muster.
(29)(a) $\lambda$ s. FRANZÖSIN(s)
(b) $\lambda u$. FRANZÖSIN $\left.{ }^{\circ} \mathrm{u}\right)$
$=\lambda \mathrm{u} . \exists \mathrm{s}[\operatorname{HOLDER}(\mathrm{u})(\mathrm{s}) \&$ FRANZÖSIN(s)]

Parsons verfolgt diese Idee aber nicht weiter, und zwar mit dem Hinweis darauf, daß sie nicht nur zu einer unnötigen Aufblähung der Theorie führt, sondern für sie auch keine überzeugende Evidenz existiert.

Tatsächlich ist der Vorschlag schon wegen des nur abgeleiteten Verständnisses der Nomina als Objektprädikate überaus fraglich. Wie bereits mit Bezug auf Chierchia (1995) festgestellt, läßt sich eine solche Sichtweise nur schlecht mit unserer Intuition in Übereinstimmung bringen. Sollte deshalb aber überhaupt der Gedanke aufgegeben werden, daß wir es auch bei nominalen Prädikativen mit Zustandsprädikaten zu tun haben?

Ein zusätzliches Argument, das für eine zustandsbasierte Analyse von DPn - wie auch von APn - in prädikativer Position spricht, findet sich bei der Betrachtung von Sätzen wie (30).

$$
\text { Fabio wurde Lehrer. }
$$

Mit (30) wird auf ein Ereignis referiert, in dessen Verlauf Fabio Gegenstand einer Veränderung war. Genauer handelt es sich um eine Veränderung derart, daß Fabio aus einem Zustand des Nicht-Lehrerseins in einen Zustand des Lehrerseins überging. Worauf also hier mit der DP Lehrer letztendlich Bezug genommen wird, ist jener Zustand, in dem sich Fabio zum Abschluß der fraglichen Veränderung befindet. Offensichtlich kann man also auch bei Sätzen

¿ Siehe beispielsweise Dölling (1998a). 
wie (30) nicht auf die Voraussetzung verzichten, daß die als Prädikativ verwendeten Ausdrükke Eigenschaften von Zuständen denotieren. ${ }^{22}$

Ich gehe davon aus, daß einem Kopulasatz wie (31) in erster Näherung eine semantische Struktur wie in (31') zugeordnet ist.

(31) Fabio war Lehrer.

(31') $\exists$ s [HOLDER(fabio)(s) \& ${ }^{+} \operatorname{LEHRER(s)]~}$

Dabei macht "," kenntlich, daß es sich beim Zustandsprädikat ${ }^{+}$LEHRER um ein gegenüber LEHRER abgeleitetes Prädikat handelt. Als Nomen hat Lehrer die semantische Repräsentation (32a) und ist damit ein Prädikat von Objekten. Nur wenn Lehrer als DP und dabei in prädikativer Position vorkommt, kann die Repräsentation (32b) angenommen werden.

(32) (a) $\lambda u$. LEHRER(u)

(b) $\lambda \mathrm{s} .{ }^{+} \operatorname{LEHRER}(\mathrm{s})$

Die genaueren Bedingungen, unter denen ein Zustandsprädikat wie (32b) zur Anwendung kommt, werde ich später formulieren. Für ein vorläufiges Verständnis reicht es aus, zu berücksichtigen, daß zwischen den Prädikaten in (32a) und (32b) die im Postulat (33) angegebene Beziehung besteht.

$$
\mathrm{N} \forall \mathrm{s}\left[{ }^{+} \operatorname{LEHRER}(\mathrm{s}) \leftrightarrow \forall \mathrm{u}[\operatorname{HOLDER}(\mathrm{u})(\mathrm{s}) \rightarrow \operatorname{LEHRER}(\mathrm{u})]\right]
$$

Demnach ist ein Zustand des Lehrerseins s dadurch charakterisiert, daß für beliebige Objekte $u$ gilt: wenn $u$ Inhaber von $s$ ist, dann gehört $u$ zur Menge der Lehrer. Wenn sich also eine Person im Zustand des Lehrerseins befindet, dann befindet sie sich in einem Zustand derart, daß jede Person, die sich in einem solchen Zustand befindet, zur Menge der Lehrer gehört. Wegen (33) ist also (32a) als eine Abkürzung für (34) anzusehen.

$$
\lambda \mathrm{s} . \forall \mathrm{u}[\operatorname{HOLDER}(\mathrm{u})(\mathrm{s}) \rightarrow \operatorname{LEHRER}(\mathrm{u})]]
$$

Damit kann zugleich (31) äquivalent, aber detaillierter durch (31') repräsentiert werden.

$$
\exists \text { s }[\operatorname{HOLDER}(\text { fabio)(s) \& } \forall \mathrm{u}[\operatorname{HOLDER}(\mathrm{u})(\mathrm{s}) \rightarrow \operatorname{LEHRER}(\mathrm{u})]]
$$

Es ist leicht zu sehen, daß sich aus (31') unmittelbar (35) logisch folgern läßt.

\section{LEHRER(fabio)}

D.h., wenn Fabio sich im Zustand des Lehrerseins befindet, dann gehört Fabio auch zur Menge der Lehrer. Was wir damit zugleich gezeigt haben, ist, daß sich der Inhalt des klassischen Verständnisses solcher Kopulasätze auf einfache Weise im Rahmen der hier entwickelten Konzeption gewinnen läßt.

An dieser Stelle ist es angebracht, einige Konsequenzen zu überdenken, die sich aus den bisherigen Annahmen über Kopulasätze für die ontologische Kategorie der Zustände ergeben. Zunächst einmal ist offensichtlich die bereits zu Beginn erwähnte Feststellung, wonach lokale

\footnotetext{
${ }^{22} \mathrm{Ob}$ sich die gegebenen Verhältnisse auch ohne die Annahme von Zuständen intuitiv angemessen erfassen lassen, muß gezeigt werden. Jedenfalls erscheint es mir zweifelhaft, daß eine Zustandsänderung als alleiniger Wechsel von entsprechenden Zeitintervallen bestimmt werden kann.
} 
Modifikatoren wie in (36a) und (36b) nicht als zustandsbezogen aufgefaßt werden können, als ein Hinweis auf die Unmöglichkeit einer räumlichen Lokalisierung von Zuständen zu deuten.

(36)(a) Anna war im Kino müde.

(b) Fabio war in Berlin Lehrer.

Mit (36a) wird nicht zu verstehen gegeben, daß der Zustand des Müdeseins von Anna im Kino lokalisiert war. Vielmehr wird dessen zeitliche Einordnung in den Zustand des Im-Kino-seins von Anna ausgedrückt. Analog wird mit (36b) der Zustand des Lehrerseins von Fabio nicht räumlich, sondern zeitlich näher spezifiziert. Dabei werden (36a) und (36b) deshalb auf diese Weise interpretiert, weil Zustände gemäß der zugrundeliegenden konzeptuellen Ontologie keine räumlichen Eigenschaften haben. ${ }^{23}$ Wenn also ein bestimmter Zustand besteht, dann ist seine Existenz nicht auf einen Ort beschränkt, sondern unabhängig von jeglichen räumlichen Bedingungen. Diese für Zustände geltenden Verhältnisse spiegeln sich parallel in Sätzen wie (37a) und (37b) wider, wo entsprechend die Zustandsverben lieben bzw. besitzen vorkommen.

(37)(a) Sebastian liebte in Italien Maria.

(b) Claudia besaß auf dem Lande ein Häuschen.

Auch hier werden die jeweils beschriebenen Zustände nicht an den durch die Adverbiale erfaßten Orten lokalisiert. Was mit in Italien bzw. auf dem Lande kenntlich gemacht wird, ist wieder eine zeitliche Einordnung oder aber eine räumliche Charakterisierung jener Objekte, die Thema der betreffenden Zustände sind. ${ }^{24}$

Wie verhält es sich nun mit der Lokalisierung von Zuständen in der Zeit? Gibt es auch Kopulasätze, die auf Zustände ohne zeitliche Bestimmungen referieren? Sätze wie (38a) und (38b) drücken so genannte ewige Wahrheiten aus und scheinen in diesem Sinne zeitlos zu sein.

(38)(a) 6 ist geradzahlig.

(b) Der Löwe ist ein Säugetier.

Eine temporale Modifikation der vorkommenden Kopula-Prädikativ-Konstruktionen, bei denen die Dauer der Zustände des Geradzahligseins der 6 bzw. des Säugetierseins der Art 'Löwe' eingeschränkt wird, ist damit aus konzeptuellen Gründen ausgeschlossen. Dennoch erfolgt natürlich über das Tempus eine, wenn auch nur formelle zeitliche Einordnung solcher Zustände. Offensichtlich ist es - im Gegensatz zu Sachverhalten etwa - eine Eigenschaft beliebiger Situationen, in der Zeit eingeordnet zu sein.

\footnotetext{
23 Siehe hierzu auch Bäuerle (1994), wo eine ähnliche Position wie hier vertreten wird.

24 Daß mit Zuständen auch räumlich nicht lokalisierbare Situationen existieren, hat eigentlich nichts Verwunderliches an sich. Mentale Ereignisse und Prozesse wie jene, auf die mit den Verben schlußfolgern oder denken, referiert werden kann, weisen nach gängiger Auffassung ebenfalls keine Lokalisierungen im Raum auf. Eine andere Frage ist, ob man mit Blick auf Verben wie warten oder schlafen und stehen oder sitzen, die lokale Adjunkte bzw. Argumente zulassen, zwischen räumlichen und nicht-räumlichen Zuständen unterscheiden sollte. Anders als beispielsweise Parsons (1990) oder Piñon (1996) denke ich, daß die häufig mit dem 'Terminus $d y$ namic state (vgl. Bach 1986, Egg 1995) charakterisierten Verben nicht als 'echte' Zustandsverben zu betrachten sind, sondern eine eigene Subsorte von Situationen bezeichnen. Zu deutlich unterscheiden sich m.E. diese beiden Verbgruppen sowohl in ihrem semantischen als auch in ihrem syntaktischen Verhalten voneinander. Abgesehen von der bereits erwähnten Eigenschaft der räumlichen Lokalisierbarkeit können 'Dynamic-state'-Verben beispielsweise außerdem in 'Pseudo-cleft'-Konstruktionen der Form Was $x$ tat, war $P$ gebraucht werden, und ihre englischen Entsprechungen lassen ohne Einschränkung die Verwendung der Progressivform zu. Aber auch wenn man sich für die Anerkennung zweier Typen von Zustandsverben entscheiden sollte, muß eine klare Differenzierung zwischen den betreffenden ontologischen Domänen vorgenommen werden. Letzteres wird weder von Parsons noch von Piñon getan.
} 
Wenden wir uns nun abschließend Kopulasätzen zu, in denen wie in (39) PPn als Prädikative auftreten. ${ }^{25}$

Hans war auf der Straße.

Analog zu den bisher analysierten Kopulasätzen drückt (39) aus, daß Hans sich im Zustand des Auf-der-Straße-seins befand. Es scheint naheliegend zu sein, dessen semantische Repräsentation einfach mit Hilfe von (39') anzugeben.

\section{$\exists \mathrm{s}[$ HOLDER(hans)(s) \& AUF(def_STRASSE)(s)]}

Wenn nicht weitere Vorkehrungen getroffen werden, besagt aber (39') nicht unbedingt, daß Hans der Inhaber eines Zustandes des Auf-der-Straße-seins war. Vielmehr kann die Formulierung auch so verstanden werden, daß Hans sich in einem Zustand s befand und s auf der StraBe lokalisiert war. ${ }^{26}$ Nach dem vorher Gesagten ist aber eine solche räumliche Lokalisierung von Zuständen gerade ausgeschlossen.

Um in Fällen wie (39) die erwünschte Adäquatheit zu erzielen, muß man die folgenden beiden Punkte berücksichtigen: Erstens ist davon auszugehen, daß lokale PPn als Prädikate unmittelbar nur auf Objekte, Ereignisse und Prozesse, nicht aber auf Zustände anwendbar sind. Zweitens existiert offensichtlich ein Mechanismus, mit dem - analog zum Vorgehen bei prädikativen DPn - für PPn, falls sie in prädikativer Position vorkommen, Prädikate für Zustände abgeleitet werden können. Bezogen auf (39) heißt das, daß die lokale PP auf der Straße zwei semantische Repräsentationen hat: eine primäre Repräsentation wie in (40a) und eine abgeleitete Repräsentation wie in (40b), die ihrerseits wiederum eine Abkürzung für ein entsprechend komplexeres Prädikat ist.

(40)(a) $\lambda \underline{x}$. AUF(def_STRASSE)( $\underline{x})$

(mit $\underline{x}$ als Variable für Objekte, Ereignisse und

(b) $\lambda \mathrm{s} .{ }^{+} \mathrm{AUF}($ def_STRASSE)(s) Prozesse)

$=\lambda \mathrm{s} . \forall \mathrm{u}[\operatorname{HOLDER}(\mathrm{u})(\mathrm{s}) \rightarrow \operatorname{AUF}($ def_STRASSE$)(\mathrm{u})]$

Satz (39) ist dann an Stelle von (39') durch (39') zu repräsentieren.

(39’') ヨs [HOLDER(hans)(s) \& ${ }^{+}$AUF(def_STRASSE)(s)]

Mit den Überlegungen dieses Abschnitts verfügen wir über Bedeutungsrepräsentationen, aus denen explizit hervorgeht, daß Kopulasätze auf Zustände referieren. Wir haben auch erste Vorstellungen über den Anteil, den zum einen die Kopula und zum anderen die jeweiligen Prädikative in die semantische Repräsentation solcher Sätze einbringen. Im weiteren gilt es zu klären, auf welche Weise sich die Bedeutungsbeiträge der einzelnen Satzkonstituenten kompositionell miteinander verknüpfen und wie in diesem Zusammenhang insbesondere die erforderlichen Anpassungen bei Prädikativen realisiert werden. Dabei wird sich zeigen, daß einige unserer bisherigen Annahmen einer Modifizierung bedürfen.

\footnotetext{
${ }^{25}$ Es ist übrigens symptomatisch, daß prädikativ gebrauchte PPn in den Überlegungen zur Unterscheidung von Stadien- und Individuenprädikaten so gut wie keine Rolle spielen.

${ }^{26}$ Tatsächlich scheint ein solches Verständnis durch Parsons $(1990,187)$ nahegelegt zu werden. Ohne Kommentar wird dort nämlich davon gesprochen wird, daß Lokative, die mit der Kopula vorkommen, für Prädikate von Zuständen stehen.
} 


\section{Kompositionelle Ableitungen für Kopulasätze}

Ich beginne wiederum mit der Betrachtung von Kopulasätzen, in denen ein adjektivisches Prädikativ vorkommt. Das hervorspringende Problem bei der Analyse eines Satzes wie (41) besteht zunächst darin, die Quelle des in seiner semantischen Repräsentation vorkommenden HOLDER-Prädikates zu bestimmen.

Peter war müde.

$$
\exists \mathrm{s}[\operatorname{HOLDER}(\text { peter)(s) \& MÜDE(s)] }
$$

Da den früheren Voraussetzungen gemäß in (41') dafür weder die Kopula noch das Adjektiv müde in Frage kommen, scheint es nur drei Optionen für die Lösung dieses Problems zu geben: Erstens kann angenommen, daß das jeweilige Subjekt die Komponente der HOLDERRelation aus dem Lexikon mitbringt. Die zweite Möglichkeit ist, sie als Bedeutungsbestandteil einer phonologisch leeren syntaktischen Konstituente zu betrachten. Und drittens kann von der Existenz einer besonderen semantischen Operation ausgegangen werden, deren Funktion das Einbringen der fraglichen Komponente ist. Wird der erste Weg eingeschlagen, so steht man dann vor der unangenehmen Konsequenz, nicht nur für Eigennamen, sondern auch für komplexe DPn wie etwa ein Mann, der Mann oder jeder Mann erklären zu müssen, über welche lexikalischen Mittel die HOLDER-Komponente eingebracht wird. ${ }^{27}$ Eine näherliegende Variante ist deshalb die zweite Option, die in Kratzer (1994) verfolgt wird. Dabei besteht nach Kratzer generell die semantische Rolle der funktionalen Kategorie 'Voice' darin, über die ihr zugeordnete Komponente einer thematischen Relation einen Landeplatz für das Subjektargument des jeweiligen Satzes zu schaffen. ${ }^{28}$ Obwohl ich diesen Vorschlag für durchaus bedenkenswert halte, entscheide ich mich aus Gründen, die unten erläutert werden, für die dritte der angegebenen Optionen. Ausgangspunkt der Überlegungen ist, daß im Verlaufe der semantischen Komposition eines Satzes wie (41) ein Konflikt zwischen der sortalen Bestimmung der Kopula-Prädikativ-Konstruktion und der des Subjektarguments zu lösen ist. So ist unter Voraussetzung von (42) als semantischer Repräsentation von müde sein eine Applikation auf Peter ausgeschlossen.

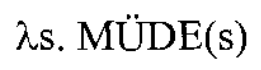

Man kann nun annehmen, daß Konflikte dieser Art durch einen Einschub des speziellen semantischen Operators (43) in die kompositionelle Ableitung aufgelöst werden. ${ }^{29}$

$$
\lambda \mathrm{P} \lambda \mathrm{u} . \exists \mathrm{s}[\mathrm{HOLDER}(\mathrm{u})(\mathrm{s}) \& \mathrm{P}(\mathrm{s})]
$$

Durch funktionale Anwendung von (43) auf (42) läßt sich dabei die in (44) angegebene abgeleitete semantische Repräsentation für müde sein gewinnen, wodurch unmittelbar die Bedingung für eine Integration des Subjektarguments erfüllt ist.

\footnotetext{
27 So nimmt Krifka (p.c.) spezielle Operationen an, durch die Eigennamen mit semantischen Repräsenationen versehen werden, in denen entsprechende Prädikate für thematische Relationen enthalten sind. Im Falle komplexer DPn werden in Krifka $(1989,1992)$ dann für die jeweiligen Determinatoren passende lexikalischsemantische Repräsentationen mit solchen Prädikaten vorausgesetzt.

${ }_{28}$ In Annäherung an die neo-Davidsonsche Variante der Ereignissemantik im Sinne von Parsons $(1980,1990)$ geht Kratzer (1994) davon aus, daß Verb(alphras)en für sich genommen keine externe Argumentstelle besitzen und daß deshalb diese erst beim Aufbau der semantischen Repräsentation des jeweiligen Satzes 'erzeugt' werden muß. (Siehe hierzu auch von Stechow 1996.) Unverständlich bleibt in diesem Zusammenhang allerdings, warum dann für Adjektive wie müde oder krank das bisherige Kratzersche Verständnis beibehalten und nicht konsequenterweise eine Repräsentation als Zustandsprädikat angenommen wird.

${ }_{29}$ Für ein solches Herangehen der sogenannten Sortenverschiebung wurde von mir erstmalig in Dölling (1992) argumentiert. Analoge Operatoren des Bedeutungstransfers werden in Nunberg (1995) vorgeschlagen. Vgl. auch Zimmermann (1999), wo in ähnlichen Fällen semantische Templates zum Einsatz kommen.
} 


$$
\lambda \text { u. } \exists \text { s [HOLDER(u)(s) \& MÜDE(s)] }
$$

Bemerkenswert ist, daß uns mit (43) zugleich ein Mittel zur Verfügung steht, um die semantische Repräsentation einer NP wie müdes Kind abzuleiten. Auch in solchen Fällen tritt ein sortaler Konflikt insofern auf, als das Nomen Kind als Prädikat von Objekten nicht unmittelbar durch das Adjektiv mïde modifiziert werden kann. Geht man entsprechend davon aus, daß zunächst mit Hilfe von Operator (43) für müde ein mit (44) identisches Objektprädikat als Repräsentation abgeleitet wird, steht einer Modfikation nichts mehr im Wege. Als semantische Repräsentation von müdes Kind erhält man dann über diesen Zwischenschritt (45).

\section{$\lambda$ u. $\exists$ s $[\operatorname{HOLDER}(\mathrm{u})(\mathrm{s}) \& \operatorname{MÜDE}(\mathrm{s})] \& \operatorname{KIND}(\mathrm{u})$}

Bevor ich weitere Anpassungsoperationen in Erwägung ziehe, ist es allerdings jetzt an der Zeit, zwei in der Darstellung bisher vernachlässigte Faktoren, nämlich Tempus und Aspekt zu berücksichtigen. Ohne weitere Diskussion sollen hier - bis auf einige Adaptionen - die in Maienborn (1998) formulierten Voraussetzungen übernommen werden. Insbesondere gehe ich davon aus, daß im gegebenen Zusammenhang für Aspekt und Tempus entsprechend die in (46) und (47) aufgeführten semantischen Strukturen angesetzt werden können, wobei in (46) mit $\subseteq$ die Möglichkeit der Fortdauer der betreffenden Situation gegeben ist. ${ }^{30}$

$$
\text { Asp; } \lambda \mathrm{P} \lambda \mathrm{t} \lambda \underline{\mathrm{s}} . \mathrm{t} \subseteq \tau(\underline{\mathrm{s}}) \& \mathrm{P}(\underline{\mathrm{s}})
$$

(mit $\mathrm{t}$ und $\underline{\mathrm{s}}$ als Variablen für für (Topik-)Zeiten bzw. Situationen und $\tau$ als Funktor 'Dauer von')

Wie aus der für Satz (48) in (48') dargestellten Ableitung zu ersehen ist, hat diese Präzisierung keinen gravierenden Einfluß auf die sortale Anpassung.

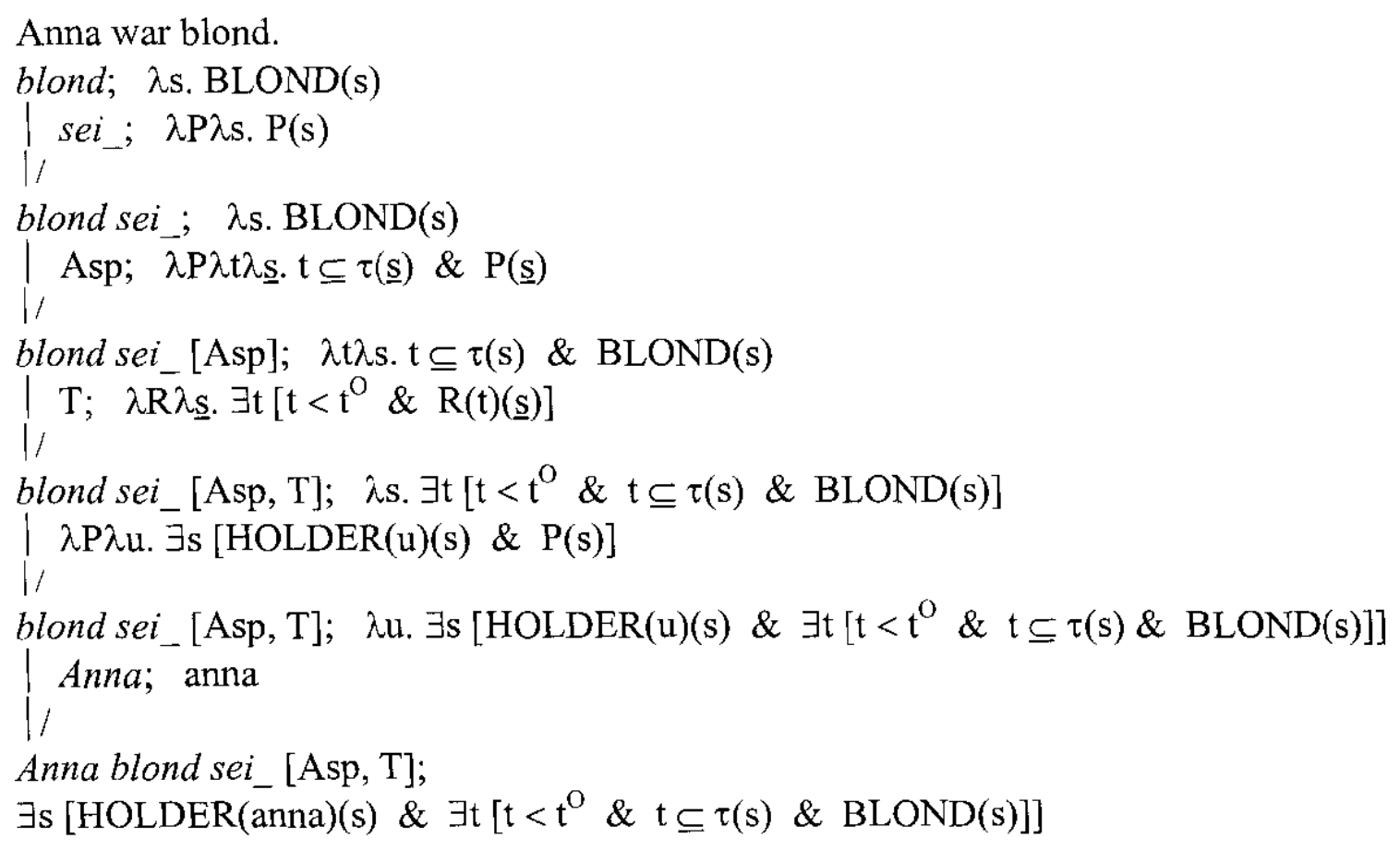

\footnotetext{
${ }^{30}$ Diese Annahmen stellen aus mehreren Gründen Vereinfachungen dar. Zum einen beinhaltet natürlich (47) nur die - für die hier betrachteten Beispielsätze einzig relevante - Repräsentation für das Präteritum. Zum anderen gehe ich davon aus, daß die Aspektkategorie - zumindest im Deutschen - als unterspezifiziert zu behandeln ist. D.h. vor allem, daß die ihr zugeordnete semantische Repräsentation an Stelle von $\subseteq$ in (46) einen Relationsparameter $A S P$ enthält, der in Abhängigkeit von kontextuellen Bedingungen durch entsprechende Werte fixiert werden kann. Und schließlich vermute ich, daß der Bezug auf Topik- und Äußerungszeit genauer über den Bezug auf eine Topik- bzw. eine Äußerungssituation realisiert wird.
} 
Das Problem der zustandsbezogenen Interpretation von nominalen oder präpositionalen Prädikativen kann nun ganz analog behandelt werden. Betrachten wir, welches Vorgehen bei einer prädikativ gebrauchten DP wie eine Ärztin denkbar wäre. Unter der Voraussetzung, daß das Nomen Ärztin den in (49) angegebenen lexikalisch-semantischen Eintrag hat, ist die DP eine Ärztin gemäß Standardauffassung zunächst in Form von (50) zu repräsentieren.

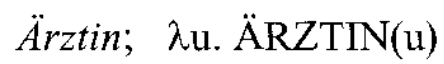

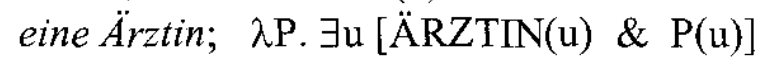

Ein Vergleich mit der für die Kopula angenommenen semantischen Repräsentation in (28) zeigt, daß (50) sowohl hinsichtlich des semantischen Typs als auch mit Blick auf die sortalen Bedingungen anpassungsbedürftig ist. $\mathrm{Da}$ die Kopula als mögliche Argumente nur Prädikate der 1. Stufe zuläßt, wird in einem ersten Schritt die semantische Repräsentation in (50) mit Hilfe des Typanpassungsoperators BE in $(51)^{31}$ in die Struktur in (52) überführt.

$$
\begin{aligned}
& \text { BE: } \lambda \wp \lambda \mathrm{x} . \wp(\lambda \mathrm{y} . \mathrm{x}=\mathrm{y}) \\
& \text { eine Arztin; } \lambda \mathrm{u} . \ddot{A} \operatorname{RZTIN}(\mathrm{u})
\end{aligned}
$$

(mit $\wp$ als Variable für Prädikate der 2. Stufe; $x$, y als sortal unbeschränkte Individuenvariablen)

Im zweiten Schritt wird der sortalen Selektionsbeschränkung der Kopula dadurch entsprochen, daß das Prädikativ eine Ärztin eine Repräsentation in Form eines Zustandsprädikats erhält. Zur Herleitung bietet es sich an, (53) als einen weiteren semantischen Anpassungsoperator vorauszusetzen.

$$
\lambda \mathrm{P} \lambda \mathrm{s} . \forall \mathrm{u}[\operatorname{HOLDER}(\mathrm{u})(\mathrm{s}) \rightarrow \mathrm{P}(\mathrm{u})]]
$$

Bei Anwendung von (53) auf die semantische Repräsentation in (52) erhält man so für die prädikativ gebrauchte DP die in (54) aufgeführte Struktur.

$$
\text { eine Ärztin; } \lambda \mathrm{s} . \forall \mathrm{u}[\mathrm{HOLDER}(\mathrm{u})(\mathrm{s}) \rightarrow \ddot{\mathrm{ARZTIN}}(\mathrm{u})]]
$$

Eine Darstellung aller Schritte, die in der kompositionellen Ableitung der semantischen Repräsentation eines Satzes wie (55) zu realisieren sind, liefert (55').

(55) Anna war eine Ärztin.

(55') Ärztin; $\lambda \mathrm{u}$. ÄRZTIN(u)

$\mid$ eine; $\lambda \mathrm{P} \lambda \mathrm{Q} . \exists \mathrm{x}[\mathrm{P}(\mathrm{x}) \& \mathrm{Q}(\mathrm{x})]$

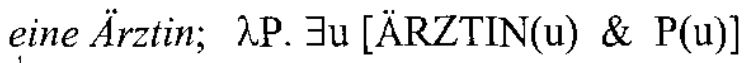

BE: $\lambda \wp \lambda \mathrm{x} \cdot \wp(\lambda \mathrm{y} \cdot \mathrm{x}=\mathrm{y})$

eine Ärztin; $\lambda \mathrm{u}$. ÄRZTIN(u)

$\lambda \mathrm{P} \lambda \mathrm{s} . \forall \mathrm{u}[\operatorname{HOLDER}(\mathrm{u})(\mathrm{s}) \rightarrow \mathrm{P}(\mathrm{u})]]$

eine Ärztin; $\lambda \mathrm{s} . \forall \mathrm{u}[\operatorname{HOLDER}(\mathrm{u})(\mathrm{s}) \rightarrow$ ÄRZTIN(u)]]

$\left.\right|_{1 /}$ sei_; $\lambda \mathrm{P} \lambda \mathrm{s} . \mathrm{P}(\mathrm{s})$

\footnotetext{
3 Siehe hierzu Partee (1992) und Dölling (1998a).
} 


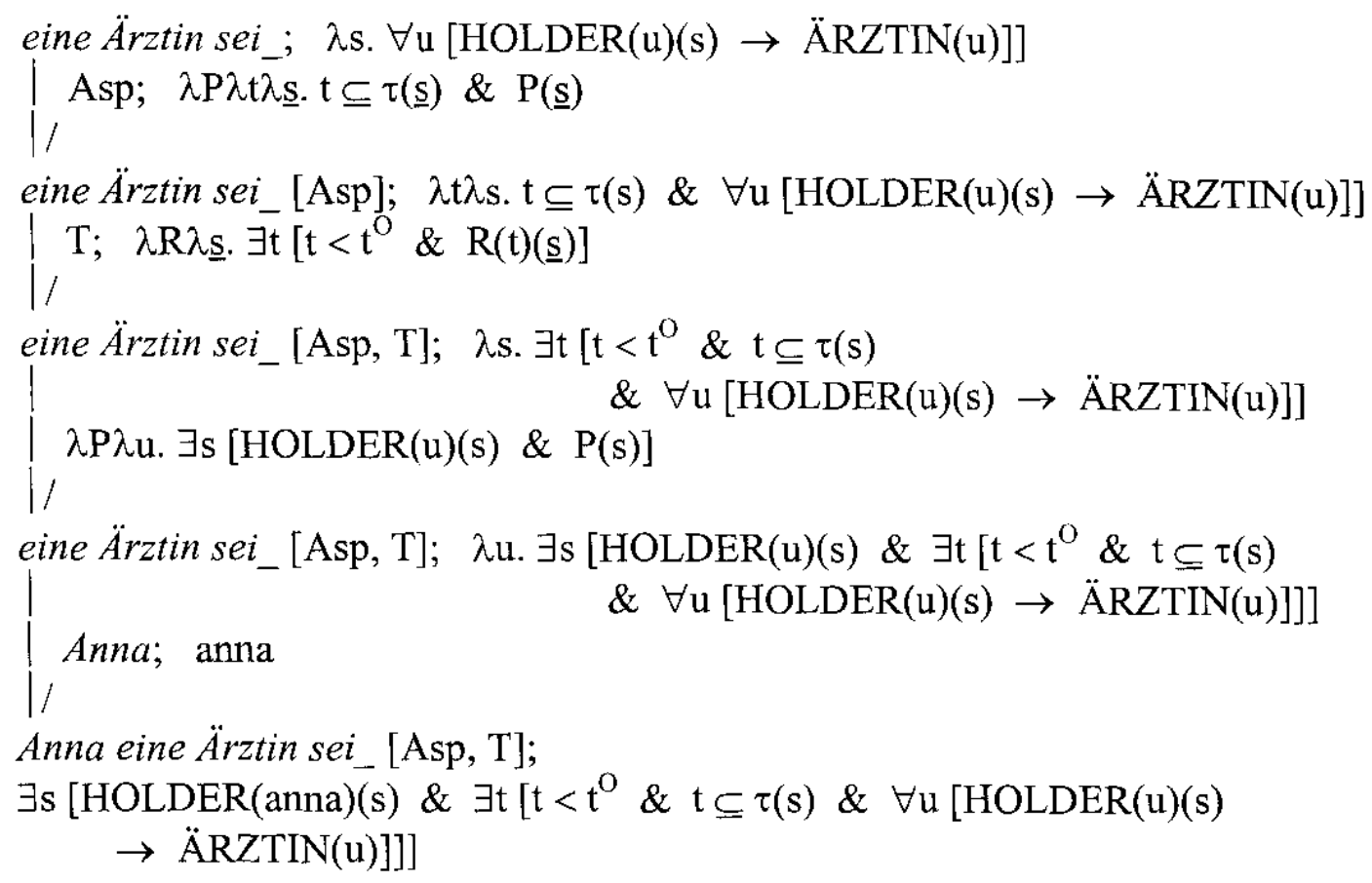

Tritt - wie in (56) - ein Adjektiv als Modifikator in der prädikativen DP auf, so muß entsprechend der Anpassungsoperator (43) zweimal zur Anwendung kommen. Als semantische Repräsentation von (56) wird dann (56') abgeleitet.

(56) Anna war eine blonde Ärztin.

(56') $\exists \mathrm{s}\left[\operatorname{HOLDER}\left(\right.\right.$ anna)(s) \& $\exists \mathrm{t}\left[\mathrm{t}<\mathrm{t}^{\mathrm{O}} \& \mathrm{t} \subseteq \tau(\mathrm{s}) \& \forall \mathrm{u}[\operatorname{HOLDER}(\mathrm{u})(\mathrm{s})\right.$ $\rightarrow \exists s^{\prime}\left[\operatorname{HOLDER}(\mathrm{u})\left(\mathrm{s}^{\prime}\right) \& \mathrm{BLOND}\left(\mathrm{s}^{\prime}\right)\right]$ \& ÄRZTIN(u)]]]]

Es ist klar, daß sich dabei die Zustände des Blondseins und des Eine-blonde-Ärztin-seins unterscheiden müssen.

Weitgehend parallel zum Fall eines Satzes mit nominalem Prädikativ werden die semantischen Repräsentationen von Kopulasätzen hergeleitet, in denen eine PP als Prädikativ fungiert. Satz (57) wird dementsprechend durch (57') repräsentiert.

(57') $\exists \mathrm{s}\left[\operatorname{HOLDER}\left(\right.\right.$ anna)(s) \& $\exists \mathrm{t}\left[\mathrm{t}<\mathrm{t}^{\circ} \& \mathrm{t} \subseteq \tau(\mathrm{s}) \& \forall \mathrm{u}[\operatorname{HOLDER}(\mathrm{u})(\mathrm{s})\right.$

$\rightarrow$ IN(def_KLINIK)(u)]]]

Nachdem wir uns davon überzeugt haben, daß sich die kompositionelle Ableitung von Kopulasätzen als Zustandsbeschreibungen im Prinzip problemlos vollziehen läßt, erhebt sich nun die Frage, ob man ganz analog auch in Fällen wie (58) oder (59) verfahren kann.

Der Knall war laut.

Die Fahrt war schnell.

Können wir insbesondere (58) und (59) so verstehen, daß sich der Knall im Zustand des Lautseins bzw. die Fahrt im Zustand des Schnellseins befanden? Da offensichtlich konzeptuell ausgeschlossen ist, daß Ereignisse oder Prozesse Inhaber von Zuständen sind, können Kopulasätze wie (58) und (59) sicher nicht auf Zustände referieren. Worauf sich solche Sätze beziehen, sind genau jene Ereignisse oder Prozesse, die mit den jeweiligen Subjekt-DPs erfaßt werden. So referieren (58) und (59) einfach auf den betreffenden Knall bzw. die betreffende 
Fahrt, von denen entsprechend ein Lautsein bzw. Schnellsein prädiziert wird. Sowohl die als Prädikative fungierenden Adjektive laut und schnell als auch die mit ihnen gebildeten Kopula-Prädikativ-Konstruktionen sind dabei Prädikate von Ereignissen bzw. Prozessen.

Eine Konsequenz dieser Beobachtungen ist, daß wir die in Abschnitt 3 formulierten Hypothesen (I) und (II) zu revidieren haben. Sie werden durch die Feststellungen (I') und (II') ersetzt, in denen in allgemeiner Form Berücksichtigung findet, daß Prädikativ-KopulaKonstruktionen nicht nur Eigenschaften von Zuständen, sondern auch der anderen Subsorten von Situationen denotieren.

(I') Beliebige Prädikative - seien sie nun nominaler, adjektivischer oder präpositionaler Art - sind Prädikate von Situationen.

(II') Die Kopula ist für eine Anwendung auf Situationsprädikate definiert und fügt nichts zu deren Bedeutung hinzu.

Entsprechend muß dann (28) durch den in (60) angeführten lexikalisch-semantischen Eintrag für die Kopula ersetzt werden.

(60) $\quad \operatorname{sein} ; \lambda \mathrm{P} \lambda \underline{\mathrm{s}} . \mathrm{P}(\underline{\mathrm{s}})$

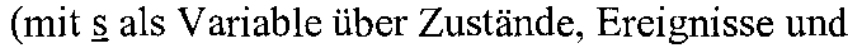
Prozesse)

Betrachten wir nun, welche semantischen Repräsentationen für Kopulasätze als Ereignis- bzw. Prozeßbeschreibungen anzunehmen sind. In (58') gebe ich die kompositionelle Ableitung für (58) wieder.

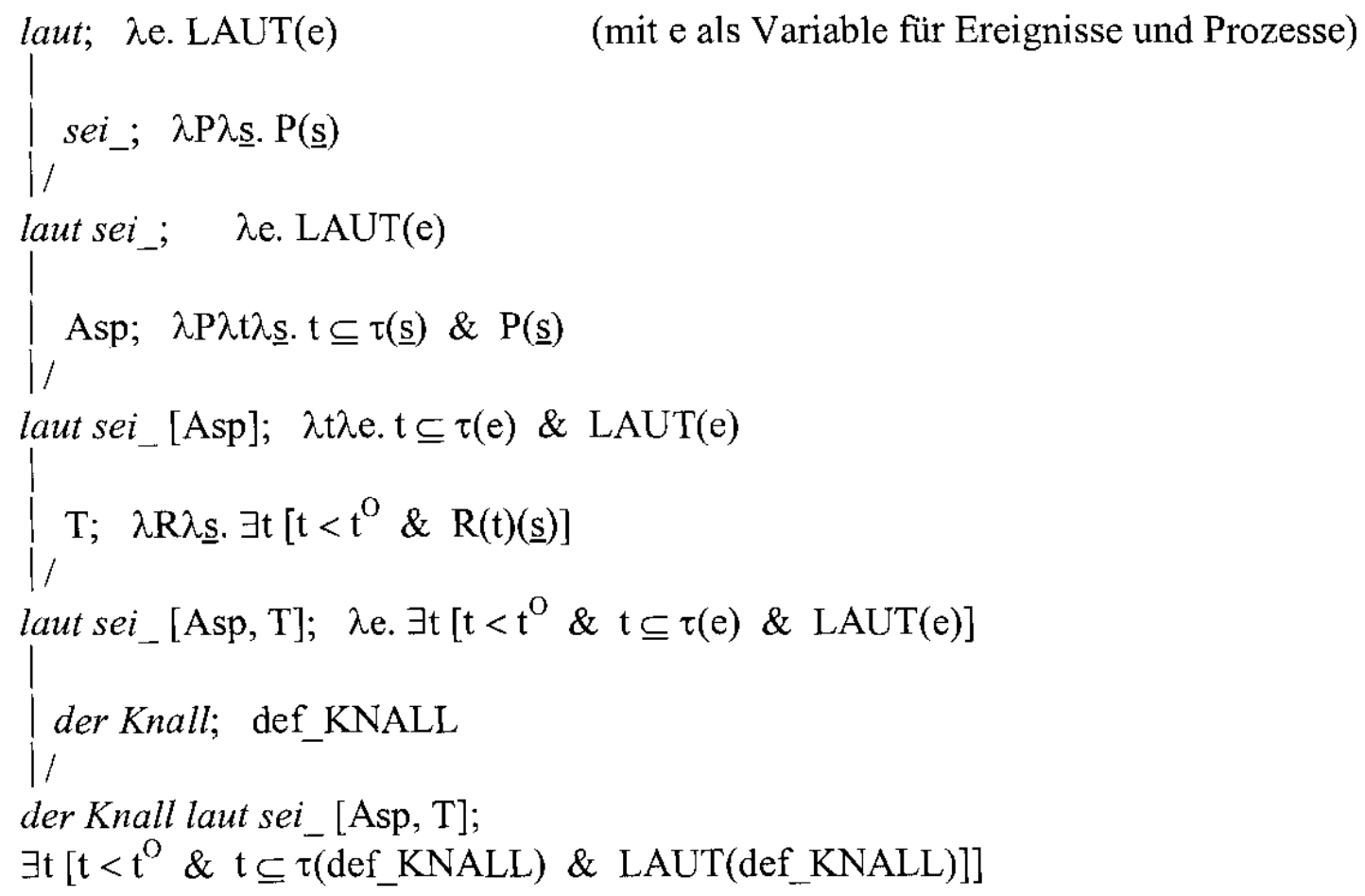

Analog sieht die Ableitung für einen Satz wie (61) aus, dessen semantische Repräsentation in (61') angegeben wird.

(61) Der Knall war auf der Straße.

(61') $\exists \mathrm{t}\left[\mathrm{t}<\mathrm{t}^{\mathrm{O}} \& \mathrm{t} \subseteq \tau(\right.$ def_KNALL) \& AUF(def_STRASSE)(def_KNALL)]] 
Der Vollständigkeit halber soll an dieser Stelle schließlich auf einen Satz wie (62) eingegangen werden.

(62) Marias Schönheit war bewundernswert.

Mit (62) wird vom betreffenden Zustand Marias natürlich nicht prädiziert, daß er sich im Zustand des Bewundernswertseins befand. Vielmehr wird dem fraglichen Zustand einfach die Eigenschaft, bewundernswert zu sein, zugeschrieben. Unter der Voraussetzung, daß die DP Marias Schönheit wie in (63) repräsentiert wird, hat dann (62) die semantische Struktur (62').

$$
\text { Marias Schönheit; ıs [SCHÖN(s) \& HOLDER(maria)(s)] }
$$

$$
\begin{aligned}
& \exists \mathrm{t}\left[\mathrm{t}<\mathrm{t}^{\mathrm{O}} \& \mathrm{t} \subseteq \tau(\mathrm{ts}[\mathrm{SCHÖN}(\mathrm{s}) \& \operatorname{HOLDER}(\text { maria })(\mathrm{s})])\right. \\
& \& \text { BEWUNDERNSWERT(is [SCHÖN(s) \& HOLDER(maria)(s)])] }
\end{aligned}
$$

Ein Problem scheinen eher Sätze wie (64) darzustellen.

Maria war laut.

Befand sich demnach Maria im Zustand des Lautseins oder handelte es sich hier um ein von Maria initiiertes Ereignis (oder einen entsprechenden Prozeß), das (bzw. der) laut war? Offensichtlich sind beide Lesarten möglich. Im ersteren Fall erhält (64) eine generische Interpretation, wobei das Adjektiv laut als ein abgeleitetes Zustandsprädikat gebraucht wird. Der so beschriebene Zustand von Maria besteht dann darin, daß sich Maria typischerweise laut verhielt (und eventuell noch so verhält). Während bisherige Analysen der Generizität nach wie vor viele Fragen offen lassen ${ }^{32}$ und deshalb hier kein Vorschlag zur Behandlung dieser Satzverwendung formuliert werden soll, leite ich in (64') die semantische Repräsentation von (64) in seiner zweiten Lesart ab.

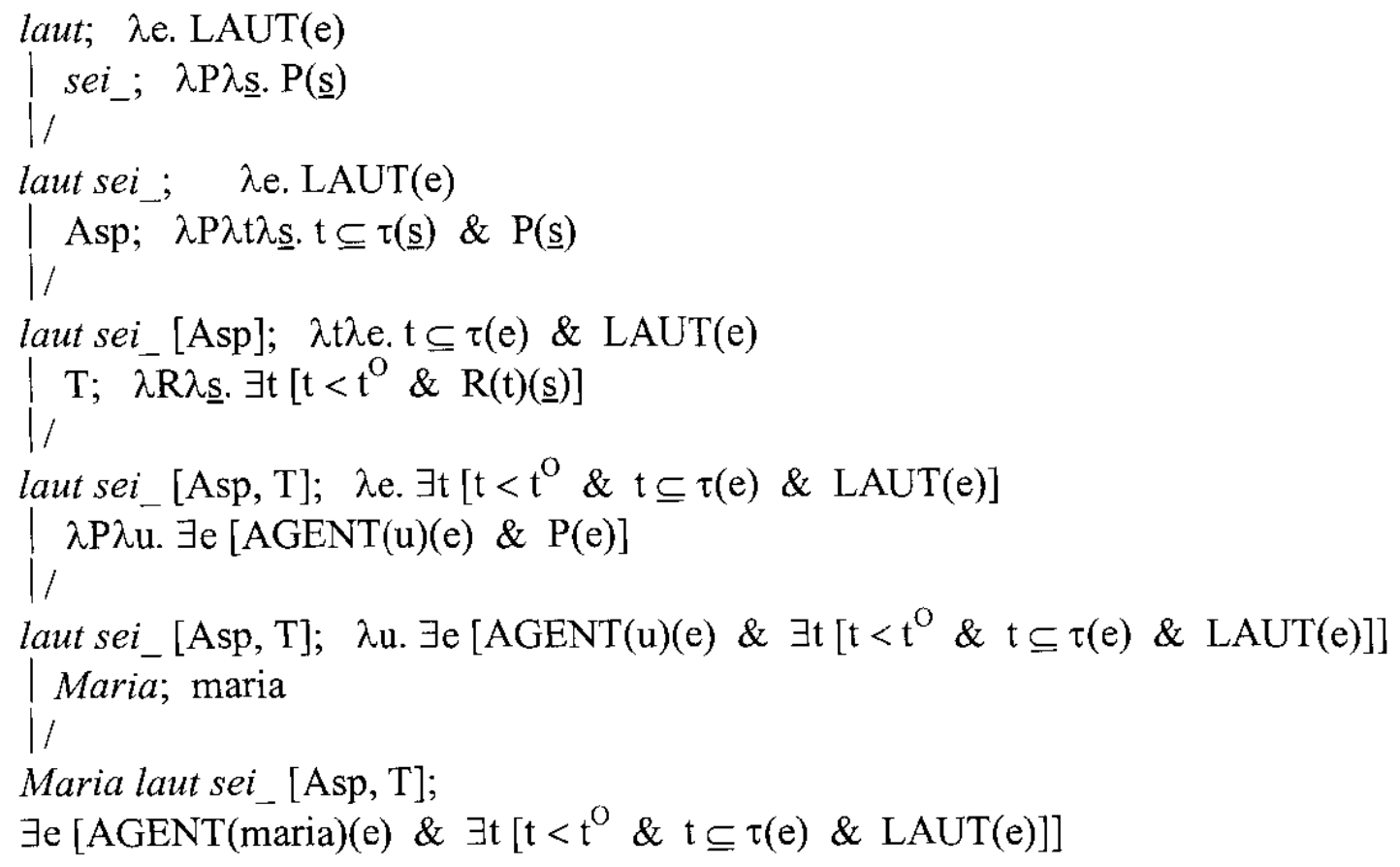

32 Siehe Krifka et al. (1995), wo Problemstellung wie auch verschiedene Analysevorschläge ausführlich diskutiert werden. 
Die Interpretation von Satz (64) als Beschreibung eines Ereignisses oder Prozesses schließt dabei in Gestalt von (65) die Anwendung eines weiteren semantischen Operators zur Auflösung eines Sortenkonfliktes ein.

$$
\lambda P \lambda u . \exists e[\operatorname{AGENT(u)(e)~\& ~P(e)]~}
$$

Mit den vorgeschlagenen sortalen Anpassungsoperationen scheinen ausreichende Mittel zur Verfügung zu stehen, um die gewünschten semantischen Repräsentationen von Kopulasätzen unter Wahrung des Kompositionalitätsprinzips der Bedeutung gewinnen zu können. Sind wir damit schon im Besitz einer adäquaten Bestimmung jener Bedeutungsphänomene, die mit der Äußerung solcher Sätze verbunden sind? Es gibt eine Reihe von Gründen, die an einer Bejahung dieser Frage zweifeln lassen. Einige von ihnen will ich nachfolgend wenigstens kurz umreißen.

\section{Semantische Form und Interpretation von Kopulasätzen}

Das Hauptproblem, mit dem die Konzeption der sortalen Anpassung semantischer Repräsentationen verbunden ist, besteht im ungeklärten Status der angenommenen Operatoren. ${ }^{33}$ Auf den ersten Blick vermitteln sie den Eindruck einer Art von 'Reparaturdienst', der immer dann zum Einsatz kommt, wenn die semantische Komposition wegen sortaler Konflikte zu scheitern droht. Soll dabei aber die Gefahr vermieden werden, daß auch in unzulässigen Fällen 'repariert' wird und dadurch absurde Resultate entstehen, müssen die Bedingungen, unter denen die fraglichen Operatoren angewandt werden dürfen, genau bestimmt sein. Eine Beschränkung der Anwendungsmöglichkeiten erfolgt sicher durch die in der Argumentstruktur vorkommenden Sortenvariablen. Damit wird allerdings nur ein Rahmen geliefert, innerhalb dessen der Gebrauch eines bestimmten Operators - etwa von (43), (53) oder (65) - letztlich allein dadurch gerechtfertigt werden kann, daß zumindest implizit auf konzeptuelles, insbesondere ontologisches Wissen zurückgegriffen wird. Dies wiederum bedeutet aber, daß die Ebene der reinen Berechnung von semantischen Repräsentationen verlassen werden muß. Fraglich ist, ob ein solches Vorgehen tatsächlich mit dem Prinzip der Kompositionalität sprachlicher Bedeutung vereinbar ist.

Die hier behandelten Formen der flexiblen Interpretation sprachlicher Ausdrücke stellen keine Ausnahmen dar. Vielmehr sind sie Instanzen eines Phänomens, das in Gestalt von Verschiebungen, Spezialisierungen und Erweiterungen der Bedeutung zu den Charakteristika des Gebrauchs von Sprache gehört. Der Versuch, alle oder wenigstens die meisten Fälle einer sortalen Anpassung über spezielle semantische Operatoren zu erfassen, würde nicht nur dazu zwingen, auf der Ebene der semantischen Repräsentation in einem weit größerem Maße zwischen Variablen unterschiedlicher Sorte zu differenzieren. Schwerwiegender ist, daß eine bloBe Auflistung von entsprechenden Anpassungsoperatoren notwendige Generalisierungen vermissen läßt und damit auch die Gefahr eines 'Wildwuchses' durch ad-hoc-Annahmen besteht.

Unter anderem um die vorangehend genannten Schwierigkeiten zu vermeiden, will ich im weiteren von einer Repräsentation der Bedeutung in unterschiedlichen Spezifikationsgraden ausgehen. Anknüpfend an die Ideen, die ich in Dölling (1997) entwickelt habe, finden dabei zwei Ebenen der Bedeutungsrepräsentation - die Semantische Form SF und die Parameter-

${ }^{33}$ Siehe zum folgenden auch Dölling (1998a, 1998b). 
fixierte Struktur PFS - Berücksichtigung. Kennzeichnend für SF als Ebene der grammatisch determinierten Bedeutung ist, daß die dort angesiedelten Repräsentationen insofern radikal unterspezifiziert sind als sie noch zu fixierende Parameter enthalten. Dagegen ist PFS jene Ebene, die im Verlaufe der abduktiven Interpretation von SF in konzeptuellem Wissen abgeleitet wird und dadurch charakterisiert ist, daß alle SF-Parameter durch spezielle Werte ersetzt sind. Es handelt sich in diesem Sinne bei PFS um eine Repräsentation der Bedeutung sprachlicher Ausdrücke, die durch kontextuelle Faktoren angereichert ist.

$\mathrm{Zu}$ den Vorzügen eines solchen Herangehens gehört, daß nun mit SF streng kompositionell aufgebaute Strukturen zur Verfügung stehen, die Variationen in der Bedeutung der jeweiligen Ausdrücke und damit deren variablen Gebrauch erlauben. Insbesondere ist es jetzt auch möglich, den Beitrag der sortalen Anpassungsoperationen zur Interpretation auf eine Weise zu rekonstruieren, daß dieser faktisch in zwei Teile zerfällt: Auf der Ebene von SF werden über sehr allgemeine Operationen, die im Verlaufe der kompositionellen Ableitung unter bestimmten Bedingungen obligatorisch zu vollziehen sind, neue SF-Parameter eingeführt. Auf der Ebene von PFS werden diese SF-Parameter durch Werte aus einem definierten Bereich derart fixiert, daß in der Konsequenz eine entsprechende Bedeutungsvariation realisiert wird. Offensichtlich gibt es berechtigten Grund zu der Vermutung, daß sich beliebige sortale Anpassungen auf eine relativ kleine Anzahl von solchen SF-Operationen gründen lassen. ${ }^{34}$

Wie leicht zu sehen ist, besitzen die oben angenommenen Operatoren (43) und (65) dieselbe Struktur. Sie - wie auch zahlreiche in anderen Zusammenhängen verwendete Operatoren der Sortenanpassung - können deshalb als Instanzen von (66) aufgefaßt werden.

$$
\lambda \mathrm{P} \lambda \mathrm{x} . \exists \mathrm{y}\left[R_{n}(\mathrm{x})(\mathrm{y}) \& \mathrm{P}(\mathrm{y})\right]
$$

Dabei handelt es sich bei $R_{n}$ um einen SF-Parameter derart, daß dessen mögliche Werte die Relation $=$ oder aber Relationen $\underline{\mathrm{R}}$ zwischen Elementen zweier ontologischen Sorten sind.

Auch (53) bietet gemeinsam mit anderen Operatoren die Möglichkeit einer verallgemeinerten Darstellung. Hierzu ist es allerdings erforderlich, zunächst eine äquivalente Umformung von (53) in (68) auf der Grundlage von Postulat (67) vorzunehmen, wobei IN' für die Relation 'befindet sich im Zustand' steht.

$$
\begin{aligned}
& \mathrm{N} \forall \mathrm{u} \forall \mathrm{s}\left[\operatorname{HOLDER}(\mathrm{u})(\mathrm{s}) \leftrightarrow \mathrm{IN}^{\prime}(\mathrm{s})(\mathrm{u})\right] \\
& \left.\lambda \mathrm{P} \lambda \mathrm{s} . \forall \mathrm{u}\left[\mathrm{IN}^{\prime}(\mathrm{s})(\mathrm{u}) \rightarrow \mathrm{P}(\mathrm{u})\right]\right]
\end{aligned}
$$

Operator (68) läßt sich dann als eine Instanz von (69) ansehen, wobei für $R_{n}$ dieselben Festlegungen wie im Falle von (66) gelten.

$$
\lambda \mathrm{P} \lambda \mathrm{x} . \forall \mathrm{y}\left[R_{n}(\mathrm{x})(\mathrm{y}) \rightarrow \mathrm{P}(\mathrm{y})\right]
$$

Mit (66) und (69) besitzen wir Schemata, die in Abhängigkeit von den jeweiligen Umständen unterschiedliche Spezifikationen von $R_{n}$ wie auch der Individuenvariablen x und y zulassen. ${ }^{35}$

\footnotetext{
34 Siehe zum folgenden die entsprechenden Vorschläge in Dölling (1992, 1997, 1998a, 1998b) und in Nunberg $(1995)$.

35 In Nunberg (1995) werden zwei Operationsschemata des Bedeutungstransfers vorgeschlagen, die im wesentlichen den unter (66) und (69) angegebenen Strukturen entsprechen.
} 
Dennoch ist es angebracht, einen weiteren Schritt in der Generalisierung zu gehen. Genauer schlage ich vor, (66) und (69) ihrerseits auf die noch grundlegendere Struktur in (70) zurückführen, wobei dann $Q_{n}$ und $C_{n}$ entsprechend als SF-Parameter für die Quantoren $\exists$ und $\forall$ bzw. für die Konnektoren $\&$ und $\rightarrow$ verwendet werden.

$$
\text { infll: } \lambda \mathrm{P} \lambda \mathrm{x} . Q_{n} \mathrm{y}\left[R_{n}(\mathrm{x})(\mathrm{y}) C_{n} \mathrm{P}(\mathrm{y})\right]
$$

In Gestalt von infl1 steht uns einer jener SF-Operatoren, oder genauer: SF-Flexive, zur Verfügung, mit denen die Primäre Semantische Form $S F_{B}$ eines Ausdrucks $\alpha$ in eine Flektierte Semantische Form $S F_{I}$ von $\alpha$ überführt und dadurch dessen Interpretationspotential erweitert wird.

Folgende Applikations- und Fixierungsbedingungen werden für infl 1 vorausgesetzt:

\section{Applikationsbedingung von infll}

Ein $\mathrm{SF}_{\mathrm{B}}(\alpha)$ vom Typ $<\mathrm{e}, \mathrm{t}>$ geht über in ein $\mathrm{SF}_{\mathrm{f}}(\alpha)$ derart, daß gilt:

$\mathrm{SF}_{\mathrm{I}}(\alpha)=\operatorname{infll}\left(\mathrm{SF}_{\mathrm{B}}(\alpha)\right)$.

\section{Fixierungsbedingungen von infll}

Ein infll $\left(\mathrm{SF}_{\mathrm{B}}(\alpha)\right)$ geht über in ein $\operatorname{PFS}(\alpha)$ derart, daß gilt:

(i) $Q_{n}$ und $C_{n}$ in infl1 $\left(\mathrm{SF}_{\mathrm{B}}(\alpha)\right)$ sind entsprechend durch $\exists$ und \& oder durch $\forall$ und $\rightarrow$ fixiert;

(ii) $R_{n}$ in infl1 $\left(\mathrm{SF}_{\mathrm{B}}(\alpha)\right)$ ist durch $=$ oder ein $\underline{\mathrm{R}}$ fixiert;

(iii) im Default-Fall sind $Q_{n}, C_{n}$ und $R_{n}$ entsprechend durch $\exists, \&$ und $=$ fixiert. $^{36}$

Nach diesen Überlegungen sind wir nunmehr in der Lage, die semantische Repräsentation eines Kopulasatzes wie (73) und dessen Interpretation genauer zu charakterisieren.

$$
\text { Hans war ein kranker Mann. }
$$

In (73') wird im ersten Schritt die SF von (73) abgeleitet. Aus Gründen der Vereinfachung lasse ich dabei die Aspekt - und die Tempuskomponente weg, ${ }^{37}$

$$
\begin{aligned}
& \text { Mann; } \mathrm{SF}_{\mathrm{B}}: \lambda \mathrm{x} \cdot \mathrm{MANN}(\mathrm{x}) \\
& \int_{/ /}^{\text {infll: }} \lambda \mathrm{P} \lambda \mathrm{x} \cdot Q_{I} \mathrm{y}\left[R_{l}(\mathrm{x})(\mathrm{y}) C_{l} \mathrm{P}(\mathrm{y})\right] \\
& \text { Mann; } \quad \mathrm{SF}_{1}: \lambda \mathrm{x} \cdot Q_{l} \mathrm{y}\left[R_{l}(\mathrm{x})(\mathrm{y}) C_{l} \operatorname{MANN}(\mathrm{y})\right]
\end{aligned}
$$

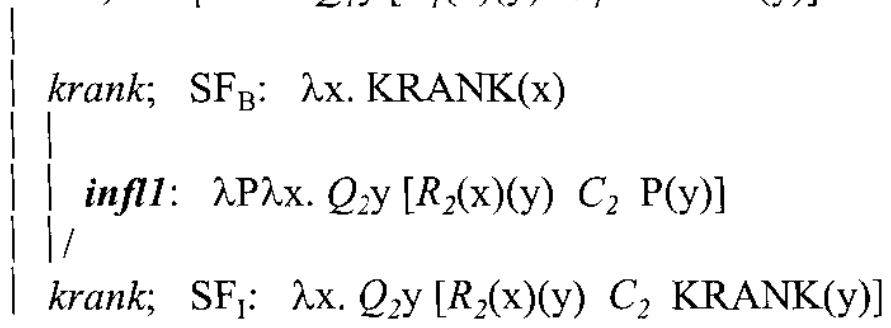

\footnotetext{
${ }^{36}$ Bedingung (iii) stellt sicher, daß in allen Fällen, in denen der jeweilige Ausdruck $\alpha$ keiner sortalen Anpassung unterzogen werden muß, der Interpretationsbeitrag von infll leer ist.

${ }^{37}$ Es sei betont, daß gerade diese beiden Komponenten für die nachfolgende Interpretation der SF von (73) eine entscheidende Rolle spielen. Nur dadurch, daß die in der Aspekt- und der Tempuskomponente vorkommenden Konstanten im konzeptuellen Wissen entsprechenden sortalen Einschränkungen unterliegen, wird überhaupt bei (73) eine zustandsbezogene Interpretation erzwungen.
} 


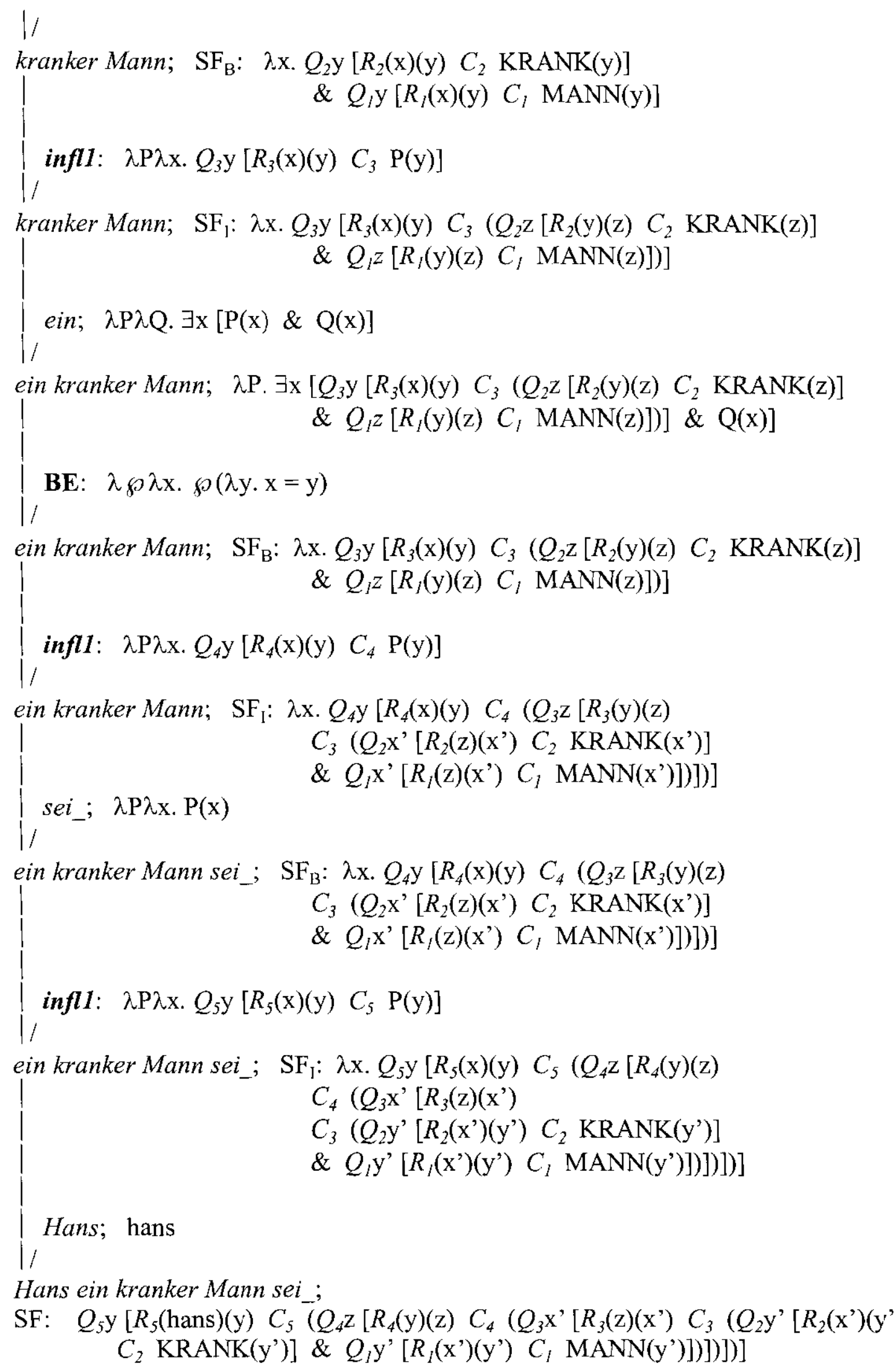

Als komputationale Bedeutungsrepräsentation ist die SF von (73) nur den SFn der im Satz vorkommenden Lexeme, seiner syntaktischen Struktur sowie den Prinzipien und Regeln der SF-Sprache verpflichtet. Insbesondere enthält die SF von (73) keine sortierten Variablen, dafür aber eine Reihe von SF-Parametern, die über die mehrfache Anwendung von infll eingebracht werden und deren Fixierung Teil der abduktiven Interpretation der SF mit Bezug auf eine konzeptuelle Wissensbasis ist. Unter der Voraussetzung, daß diese Wissensbasis Postula- 
te enthält, durch die unter anderem die Konstanten ,hans“, „KRANK“, „MANN“, „HOLDER“, und „IN"“ entsprechend charakterisiert werden ${ }^{38}$, und ein passender pragmatischer Interpretationsrahmen existiert, läßt sich im nächsten Schritt die PFS von (73) herleiten.

In (73') gebe ich zunächst die PFS von (73) in der Form an, die sie als Ergebnis von Parameterfixierungen erhält. Durch äquivalente Umformungen - unter anderem auch aufgrund von Postulat (67) - wird dann diese Struktur weiter vereinfacht.

$$
\begin{gathered}
\text { PFS: } \exists y\left[\operatorname { H O L D E R } ( \text { hans } ) ( y ) \& \left(\forall \mathrm { z } \left[\operatorname { I N } ^ { \prime } ( \mathrm { y } ) ( \mathrm { z } ) \rightarrow \left(\exists \mathrm { x } ^ { \prime } \left[=(\mathrm{z})\left(\mathrm{x}^{\prime}\right)\right.\right.\right.\right.\right. \\
\&\left(\exists \mathrm{y}^{\prime}\left[\operatorname{HOLDER}\left(\mathrm{x}^{\prime}\right)\left(\mathrm{y}^{\prime}\right) \& \operatorname{KRANK}\left(\mathrm{y}^{\prime}\right)\right]\right. \\
\left.\left.\left.\left.\left.\left.\& \exists \mathrm{y}^{\prime}\left[=\left(\mathrm{x}^{\prime}\right)\left(\mathrm{y}^{\prime}\right) \& \operatorname{MANN}\left(\mathrm{y}^{\prime}\right)\right]\right)\right]\right)\right]\right)\right] \\
\Leftrightarrow \exists \mathrm{x}\left[\operatorname { H O L D E R } ( \text { hans } ) ( \mathrm { x } ) \& \forall \mathrm { y } \left[\operatorname{IN}^{\prime}(\mathrm{x})(\mathrm{y}) \rightarrow\right.\right. \\
\left.\left.\&\left(\exists \mathrm{x}^{\prime}\left[\operatorname{HOLDER}(\mathrm{y})\left(\mathrm{x}^{\prime}\right) \& \operatorname{KRANK}\left(\mathrm{x}^{\prime}\right)\right] \& \operatorname{MANN}(\mathrm{y})\right]\right)\right] \\
\Leftrightarrow \exists \mathrm{x}[\operatorname{HOLDER}(\operatorname{hans})(\mathrm{x}) \& \forall \mathrm{y}[\operatorname{HOLDER}(\mathrm{y})(\mathrm{x}) \rightarrow \\
\left.\left.\& \exists \mathrm{x}^{\prime}\left[\operatorname{HOLDER}(\mathrm{y})\left(\mathrm{x}^{\prime}\right) \& \operatorname{KRANK}\left(\mathrm{x}^{\prime}\right)\right] \& \operatorname{MANN}(\mathrm{y})\right]\right]
\end{gathered}
$$

Zur Verdeutlichung der Möglichkeiten, die sich mit der vorgeschlagenen Trennung von SFAbleitung und SF-Interpretation für die Analyse von Kopulasätzen ergeben, seien zwei weitere Beispiele betrachtet. Ich beschränke mich dabei jeweils auf die Angabe der - durch Weglassung der Aspekt- und der Tempuskomponente vereinfachten - SFn sowie der aus ihnen hergeleiteten PFSn. Die notwendigen konzeptuellen Bedingungen der Interpretation werden wiederum vorausgesetzt.

Ich beginne mit einer Betrachtung von Satz (59), der nachfolgend unter (74) wiederholt wird.

$$
\text { Die Fahrt war schnell. }
$$

$$
\begin{gathered}
\mathrm{SF}: \quad Q_{4} \mathrm{y}\left[R _ { 4 } ( \text { def_FAHRT } ) ( \mathrm { y } ) C _ { 4 } \left(Q _ { 3 } \mathrm { z } \left[R _ { 3 } ( \mathrm { y } ) ( \mathrm { z } ) C _ { 3 } \left(Q _ { 2 } \mathrm { x } ^ { \prime } \left[R_{2}(\mathrm{z})\left(\mathrm{x}^{\prime}\right)\right.\right.\right.\right.\right. \\
\left.\left.\left.\left.\left.C_{2}\left(Q_{1} \mathrm{y}^{\prime}\left[R_{l}\left(\mathrm{x}^{\prime}\right)\left(\mathrm{y}^{\prime}\right) C_{l} \operatorname{SCHNELL}\left(\mathrm{y}^{\prime}\right)\right]\right)\right]\right)\right]\right)\right]
\end{gathered}
$$

$$
\begin{aligned}
\text { PFS: } & \exists y\left[=(\text { def_FAHRT })(y) \&\left(\exists z \left[=(y)(z) \&\left(\exists x ^ { \prime } \left[=(z)\left(x^{\prime}\right)\right.\right.\right.\right.\right. \\
& \left.\left.\left.\left.\left.\&\left(\exists y^{\prime}\left[=\left(x^{\prime}\right)\left(y^{\prime}\right) \& \operatorname{SCHNELL}\left(y^{\prime}\right)\right]\right)\right]\right)\right]\right)\right] \\
\Leftrightarrow & \exists y[=(\text { def_FAHRT })(y) \& \operatorname{SCHNELL}(y)] \\
\Leftrightarrow & \text { SCHNELL }(\text { def_FAHRT })
\end{aligned}
$$

Analog zum bereits früher analysierten Satz (58) haben wir es mit einem Kopulasatz zu tun, in dem einfach über ein Ereignis oder einen Prozeß prädiziert wird. Anders verhält es sich aber bei Satz (75).

$$
\text { Das Auto war schnell. }
$$

$$
\begin{aligned}
& \text { SF: } \quad Q_{4} \mathrm{y}\left[R _ { 4 } \left(\text { def_AUTO)(y) } C _ { 4 } \left(Q _ { 3 } \mathrm { z } \left[R _ { 3 } ( \mathrm { y } ) ( \mathrm { z } ) C _ { 3 } \left(Q _ { 2 } \mathrm { x } ^ { \prime } \left[R_{2}(\mathrm{z})\left(\mathrm{x}^{\prime}\right)\right.\right.\right.\right.\right.\right. \\
& \left.\left.\left.\left.\left.C_{2}\left(Q_{1} \mathrm{y}^{\prime}\left[R_{I}\left(\mathrm{x}^{\prime}\right)\left(\mathrm{y}^{\prime}\right) C_{I} \operatorname{SCHNELL}\left(\mathrm{y}^{\prime}\right)\right]\right)\right]\right)\right]\right)\right]
\end{aligned}
$$

In seiner nicht-generischen Interpretation hat (75) die in (75') aufgeführte PFS.

\footnotetext{
${ }^{38}$ Eine detaillierte Diskussion der konzeptuellen Grundlagen kann hier der gebotenen Kürze wegen nicht erfolgen. $\mathrm{Zu}$ den erforderlichen Postulaten gehören insbesondere solche, durch die die fraglichen Konstanten sortal festgelegt sind. Siehe auch den in Anmerkung 37 enthaltenen Hinweis auf die Funktion weiterer Konstanten bei der Fixierung der SF-Parameter von (73).
} 


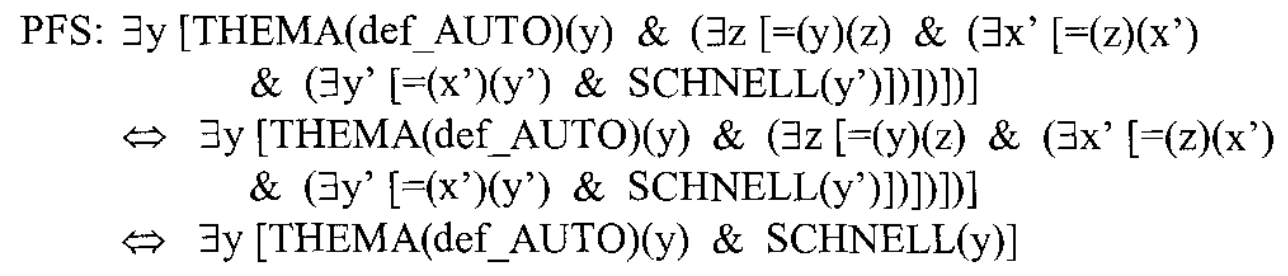

Aus (75') ist zu entnehmen, daß sich eine Äußerung von (75) ebenso wie im Falle von (64) auf ein Ereignis oder einen Prozeß bezieht, in das bzw. in den das durch die Subjekt-DP denotierte Objekt als Partizipant involviert ist. Im Gegensatz zu (64) übernimmt es dabei aber nicht die Rolle des Agens, sondern die des Themas.

\section{Schlußbemerkungen}

Im Beitrag habe ich gezeigt, daß Kopulasätze - soweit sie sich nicht auf Ereignisse oder Prozesse beziehen - als Beschreibungen von Zuständen aufzufassen sind. Dabei ist zunächst demonstriert worden, daß bisherige Versuche, die Zustandsreferenz in der semantischen Analyse umzusetzen, zu unterschiedlichen Schwierigkeiten führen. Das hier vorgestellte Konzept geht davon aus, daß es sich bei beliebigen Prädikativen letztendlich um Prädikate von Situationen handelt und die Kopula nichts zu deren Bedeutung hinzufügt. Von grundlegender Wichtigkeit ist dabei die Annahme von sortalen Anpassungen, durch die insbesondere nominale und präpositionale Prädikative, die in ihrer primären Bedeutung Eigenschaften von Objekten denotieren, einer entsprechenden Uminterpretation unterzogen werden. Abschließend ist eine Generalisierung des Herangehens vorgestellt worden. Ein Vorzug der in diesem Zusammenhang realisierten Rekonstruktion ist, daß damit auch in der Analyse von Kopulasätzen eine klare Differenzierung zwischen einem streng kompositionell strukturierten Bedeutungsanteil und einem durch konzeptuelles Wissen lizensierten Interpretationsanteil vorgenommen werden kann.

\section{Literaturverzeichnis}

Bach, Emmon (1986): 'The Algebra of Events'. In: Linguistics and Philosophy 9. 5-16

Bäuerle, Rainer (1994): 'Zustand - Prozeß - Ereignis. Zur Kategorisierung von Verb(al-phras)en.' In: Wuppertaler Arbeitspapiere zur Sprachwissenschaft. Nr. 10. Bergische Universität - Gesamthochschule Wuppertal. 132

Bierwisch, Manfred (1988): 'On the Grammar of Local Prepositions'. In: M. Bierwisch, W. Motsch \& I. Zimmermann (eds.): Syntax, Semantik und Lexikon. Berlin: Akademie-Verlag. 1-65

Chierchia, Gennaro (1995): 'Individual-level Predicates as Inherent Generics'. In: G. Carlson \& J. Pelletier (eds.): The Generic Book. Chicago: University of Chicago Press. 176-223

Dölling, Johannes (1992): 'Flexible Interpretationen durch Sortenverschiebung'. In: I. Zimmermann \& A. Strigin (Hrsg.): Fügungspotenzen. Berlin: Akademie Verlag. 23-62

- (1997): Semantic Form and Abductive Fixation of Parameters. In: R. van der Sandt, R. Blutner \& M. Bierwisch (eds.): From Underspecification to Interpretation. Working Papers of the Institute for Logic and Linguistics, Nr. 29, IBM Deutschland. Heidelberg. 113-139

- (1998a): 'Ist die Kopula mehrdeutig? Anmerkungen zu einem Vorurteil'. In: U. Scheffler \& K. Wuttich (Hrsg.): Terminigebrauch und Folgebeziehung. Berlin: Logos. 5-24

- (1998b): 'Modifikation von Resultatszuständen und lexikalisch-semantische Repräsentationen.' In: P. Ludewig \& B. Geurts (Hrsg.): Lexikalische Semantik aus kognitiver Sicht-Perspektiven im Spannungsfeld linguistischer und psychologischer Modellierungen. Tübingen: Narr. 173-206

Eckardt, Regine (1998a): Adverbs, Events and Other Things. Issues in the Semantics of Manner. Tübingen: Niemeyer

- (1998b): 'Event Semantics'. Ms. To appear in: Linguistische Berichte

Egg, Markus (1995): Aktionsart und Kompositionalität. Berlin: Akademie Verlag

Herweg, Michael (1991): 'Perfective and Imperfective Aspect in the Theory of Events and States'. In: Linguistics 29. 363-402 
Higginbotham, James \& Ramchand, Gillian (1996): 'The Stage-Level/Individual-Level Distinction and the Mapping Hypothesis'. University of Oxford (Ms.)

Jacobs, Joachim (1995): Wieviel Syntax braucht die Semantik? Möglichkeiten und Grenzen einer sparsamen Theorie der Bedeutungskomposition. Arbeiten des SFB 282 „Theorie des Lexikons“, Nr. 73. Universität Wuppertal

Jäger, Gerhard (1999): 'Stage Levels, States, and the Semantics of the Copula' (in diesem Band)

Kamp, Hans \& Reyle, Uwe (1993): From Discourse to Logic. Introduction to Modeltheoretic Semantics of Natural Language, Formal Semantics and Discourse Representation Theory. Dordrecht: Kluwer

Katz, Graham (1995): Stativity, Genericity, and Temporal Reference. Rochester (Ph.D. Dissertation)

Kratzer, Angelika (1989): 'Stage-Level and Individual-Level Predicates'. In: Papers on Quantification. NSF Grant Report. University of Massachusetts Amherst

- (1994): The Event Argument and the Semantics of Voice. University of Massachusetts Amherst (Ms.)

Krifka, Manfred (1989): Nominalreferenz und Zeitkonstitution. Zur Semantik von Massentermen, Pluraltermen und Aspektklassen. München: Fink Verlag

- (1992): 'Thematic Relations as Links between Nominal Reference and Temporal Constitution'. In: I. Sag \& A. Szabolcsi (eds.): Lexical Matters. CSLI Lecture Notes Series. Stanford: CSLI Publications. 29-53

Krifka, Manfred / Pelletier, Francis Jeffry / Carlson, Gregory N. / ter Meulen, Alice / Link, Godehard \& Chierchia, Gennaro (1995): 'Genericity. An Introduction'. In: G. N. Carlson \& F. J. Pelletier (eds.): The Generic Book. Chicago: University of Chicago Press. 1-124

Maienborn, Claudia (1996): Situation und Lokation. Die Bedeutung lokaler Adjunkte von Verbalprojektionen. Tübingen: Stauffenburg

- (1998): 'The Grammar and Pragmatics of Locative Modifiers'. Humboldt-Universität zu Berlin (Ms.)

- (1999): 'Situationsbezug und die Stadien/Individuen-Distinktion bei Kopula-Prädikativ-Konstruktionen' (in diesem Band)

Musan, Renate (1997): 'Tense, Predicates, and Lifetime Effects'. In: Natural Language Semantics 5. 271-301

Nunberg, Geoffrey (1995): 'Transfers of Meaning'. In: Journal of Semantics 12. 109-132

Parsons, Terence (1980): 'Modifiers and Quantifiers in Natural Language'. In: Canadian Journal of Philosophy VI, 29-60

- (1990): Events in the Semantics of English: A Study in Subatomic Semantics. Cambridge: MIT Press

- (1995): 'Thematic Relations and Arguments'. In: Linguistic Inquiry 26. 635-662

Partee, Barbara (1992): 'Syntactic Categories and Semantic Types'. In: M. Rosner \& R. Johnson (eds.): Computational Linguistics and Formal Semantics. Cambridge: Cambridge University Press. 97-126

Piñon, Christopher (1996): An Ontology for Event Semantics. Stanford (Ph.D. Dissertation)

Simons, Peter (1987): Parts. A Study in Ontology. Oxford: Clarendon Press

von Stechow, Arnim (1996): 'The Different Readings of Wieder 'Again': A Structural Account'. In: Journal of Semantics $13.87-138$

Zimmermann, Ilse (1996): 'Substantivgruppen mit einem Adjektivabstraktum als Kern'. In: Wuppertaler Arbeitspapiere zur Sprachwissenschaft. Nr. 12. Bergische Universität - Gesamthochschule Wuppertal. 189-208

- (1999): Partizip II-Konstruktionen des Deutschen als Modifikatoren (in diesem Band) 